\title{
Plant homeodomain finger protein 2 promotes bone formation by demethylating and activating Runx2 for osteoblast differentiation
}

Hye-Jin Kim ${ }^{1}$, Jong-Wan Park ${ }^{1,2,3}$, Kyoung-Hwa Lee ${ }^{1,2}$, Haejin Yoon ${ }^{1}$, Dong Hoon Shin ${ }^{1,2}$, Uk-Il Ju ${ }^{1}$, Seung Hyeok Seok ${ }^{4}$, Seung Hyeon Lim ${ }^{5}$, Zang Hee Lee ${ }^{6}$, Hong-Hee Kim ${ }^{6}$, Yang-Sook Chun ${ }^{1,2}$

${ }^{I}$ Department of Biomedical Sciences, Seoul National University College of Medicine, Seoul 110-799, Republic of Korea; ${ }^{2}$ Ischemicl Hypoxic Disease Institute, Seoul National University College of Medicine, Seoul 110-799, Republic of Korea; ${ }^{3}$ Cancer Research Institute, Seoul National University College of Medicine, Seoul 110-799, Republic of Korea $;{ }^{4}$ Department of Microbiology and Immunology, Seoul National University College of Medicine, Seoul 110-799, Republic of Korea, ${ }^{5}$ Institute for Experimental Animals, Seoul National University College of Medicine, Seoul 110-799, Republic of Korea; ${ }^{6}$ Department of Cell and Developmental Biology, Seoul National University School of Dentistry, Seoul 110-749, Republic of Korea

Plant homeodomain finger protein 2 (PHF2), which contains a plant homeodomain and a Jumonji-C domain, is an epigenetic regulator that demethylates lysine 9 in histone 3 (H3K9me2). On the other hand, runt-related transcription factor 2 (Runx2) plays essential roles in bone development and regeneration. Given previous reports that the PHF2 mutation can cause dwarfism in mice and that PHF2 expression is correlated with that of Runx2 in differentiating thymocytes, we investigated whether PHF2 regulates Runx2-mediated bone formation. Overexpression of PHF2 facilitated bone development in newborn mice, and viral shRNA-mediated knockdown of PHF2 delayed calvarial bone regeneration in adult rats. In primary osteoblasts and $\mathrm{C} 2 \mathrm{C} 12$ precursor cells, PHF2 enhances osteoblast differentiation by demethylating Runx2, while suppressor of variegation 3-9 homolog 1 (SUV39H1) inhibits bone formation by methylating it. The PHF2-Runx2 interaction is mediated by the Jumonji-C and Runt domains of the two proteins, respectively. The interaction between Runx2 and osteocalcin promoter is regulated by the methylation status of Runx2, i.e., the interaction is augmented when Runx2 is demethylated. Our results suggest that SUV39H1 and PHF2 reciprocally regulate osteoblast differentiation by modulating Runx2-driven transcription at the post-translational level. This study may provide a theoretical basis for the development of new therapeutic modalities for patients with impaired bone development or delayed fracture healing.

Keywords: PHF2; SUV39H1; Runx2; lysine methylation; osteoblast differentiation

Cell Research (2014) 24:1231-1249. doi:10.1038/cr.2014.127; published online 26 September 2014

\section{Introduction}

Plant homeodomain finger protein 2 (PHF2) belongs to the lysine demethylase 7 family in the Jumonji-C superfamily because it contains both a plant homeodomain (PHD) and a Jumonji-C (JmjC) domain at its N-terminus. The PHD recognizes methylated lysine residues, and the

Correspondence: Jong-Wan Park ${ }^{\mathrm{a}}$, Yang-Sook Chun ${ }^{\mathrm{b}}$

${ }^{a}$ E-mail: parkjw@snu.ac.kr

bE-mail: chunys@snu.ac.kr

Received 5 January 2014; revised 9 July 2014; accepted 5 August 2014; published online 26 September 2014
JmjC domain catalyzes the removal of methyl groups from lysine residues by using $\mathrm{Fe}^{2+}$ and 2-oxoglutarate as cofactors [1]. Functionally, PHF2 has been identified as a histone demethylase that reverses dimethylation of histone $\mathrm{H} 3$ at lysine 9 (H3K9me2). H3K9 methylation is regarded as transcription-repressive because of its association with heterochromatin assembly [2]. However, H3K9 methylation also occurs at active genes in mammalian chromatin and is associated with transcription elongation [3]. Thus, the precise consequences of PHF2-mediated H3K9 demethylation on gene expression remain unclear. Nonetheless, PHF2 is generally believed to act as a transcriptional coactivator by reversing histone methylation. For example, 
PHF2 has been found to participate in energy homeostasis by activating HNF4 and FXR and to induce adipocyte differentiation by activating CEBP $\alpha[4,5]$. In addition, PHF2 has been shown to promote inflammatory responses by inducing the expression of $\mathrm{NF}_{-} \kappa \mathrm{B}$, which then enhances expression of proinflammatory genes [6]. Given that H3K9 modification is widely involved in epigenetic regulation, PHF2 likely participates in diverse biological processes beyond those mentioned above.

Runt-related transcription factor 2 (Runx2) plays essential roles in osteoblast differentiation during bone development and remodeling. Runx 2 binds to a conserved promoter sequence (R/TACCRCA) and transactivates genes encoding osteogenic proteins such as collagen $\alpha 1$, osteopontin, bone sialoprotein (BSP), and osteocalcin (OCN) [7,8]. Runx2 is post-translationally activated during the early stage of osteoblast differentiation. Runx2 is activated by phosphorylation at serine or threonine residues through the MAPK-ERK pathway but inactivated by phosphorylation at other residues by GSK3 $\beta$ and CDK4 [9, 10]. In addition, acetylation is involved in Runx 2 signaling. For instance, p300 and p300/CBP-associated factor have been reported to enhance the transcriptional activity of Runx 2 by acetylating Runx 2 at lysine residues as well as to stabilize Runx 2 by inhibiting Runx 2 ubiquitination by Smurf1 [11, 12]. In contrast, the deacetylase HDAC3 is known to interact with Runx2 to repress the expression of Runx2 target genes [13]. Similarly, several members of the class II HDAC family have been shown to act as repressors in regulating the expression of Runx2-driven genes [14]. In addition, Runx 2 gene expression is positively regulated by the JmjC-domain-containing histone demethylase Jmjd3, which demethylates H3K27me3 at the promoter region of Runx2 [15].

The current study was motivated by two reports linking PHF2 to bone development and Runx2 signaling, respectively. Hansen et al. [16] observed that mutation of the PHF2 gene causes dwarfism, following random mutagenesis by retroviral transduction of gene-trap vectors into mouse embryonic stem cells. Zhao et al. [17] found that the expression of PHF2 is strongly related to that of Runx 2 across all stages of thymocyte differentiation. Given these two studies, we hypothesized that PHF2 might interact with Runx2 to regulate bone formation. Indeed, the results of our in vitro and in vivo experiments indicate that PHF2 plays an essential role in bone formation as a positive regulator of Runx2 activity, suggesting that PHF2 could be a novel target for enhancing bone formation.

\section{Results}

Bone development is facilitated in PHF2 transgenic mice To examine the role of PHF2 in bone development,
PHF2 transgenic ( $\mathrm{t} / \mathrm{g}$ ) heterozygous mice were generated, and their whole skeletons on postnatal day 1 were compared with those of their wild-type littermates. Bone and cartilage were co-stained using Alizarin red and Alcian blue, respectively. Alizarin red staining was denser in PHF2-t/g mice than in wild-type mice, whereas Alcian blue staining intensity did not differ significantly (Figure 1A, left panel). A close examination of three skeletal regions revealed that they were better developed in PHF2-t/g mice than in wild-type mice (Figure 1A, right panel). However, the limb and spine lengths were similar between the two groups (Supplementary information, Figure S1), suggesting that bone growth in length due to endochondral ossification is not affected by PHF2 overexpression. Following confirmation of PHF2 overexpression in the calvarial bones of PHF2-t/g mice by western blotting and immunostaining (Figure 1B), calvarial bone area and density of PHF2-t/g mice were compared with those of wild-type mice by micro-CT. PHF2-t/g mice displayed larger and denser bones than wild-type mice (Figure 1C). To analyze the extent of new bone formation in fetal mice, we first stained fetal bones with Calcein green for 2 days and further stained them with Alizalin red for another 2 days, through intraperitoneal injection of these dyes, into pregnant mice (see details in Materials and Methods). The area of new bone formed over 2 days (revealed by red staining in the absence of green) was significantly larger in PHF2-t/g mice (Figure 1D). Moreover, bone formation was verified using von Kossa staining (dark purple color generated by silver deposition to calcium phosphate) and OCN immunofluorescence (Figure 1E). We next examined osteoblast differentiation in calvarial bones by measuring the mRNA levels of osteogenic genes, which revealed higher expression of these genes in PHF2-t/g calvarias (Figure 1F). In contrast to newborn mice, the gross appearance and skeletal structure of 3-month-old mice did not differ significantly between PHF2-t/g and wild-type groups (Supplementary information, Figure S2). These results suggest that PHF2 overexpression facilitates bone development during the neonatal stage, but does not affect bone growth afterwards.

\section{PHF2 promotes osteoblast differentiation}

Given the earlier onset of bone development observed in PHF2-t/g mice, the involvement of PHF2 in osteoblast differentiation, a critical step in bone development, was examined. Primary osteoblasts were isolated from calvarias of PHF2-t/g and wild-type mice on postnatal day 1 and treated with BMP2 to induce differentiation. Indeed, western blotting revealed that PHF2-t/g osteoblasts showed a higher expression level of PHF2 than wildtype ones (Figure 2A). In addition, PHF2 seems to be expressed in parallel with Runx 2 because the expression of 
both proteins was induced during the early stage of differentiation but decreased after day 5 of differentiation in wild-type osteoblasts (Figure 2A). Immunofluorescence analysis revealed that PHF2 and Runx2 were co-local-

A

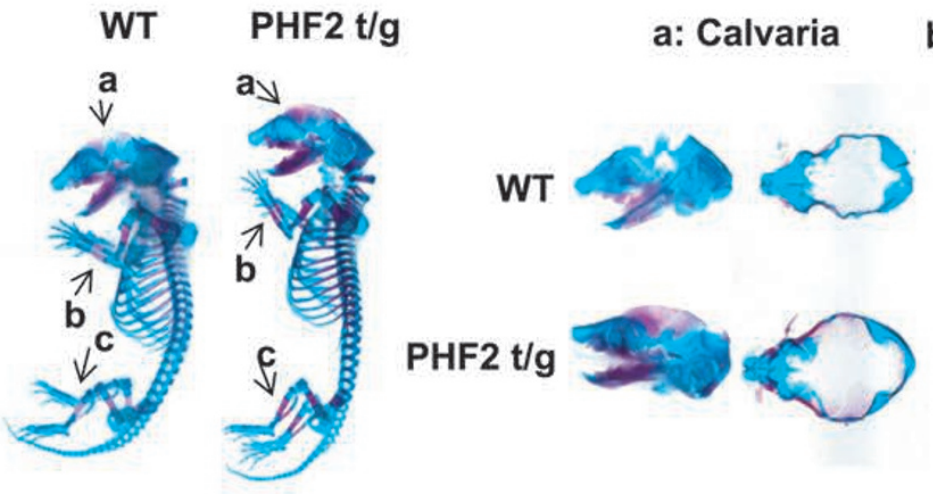

b: Forelimb c: Hindlimb

B

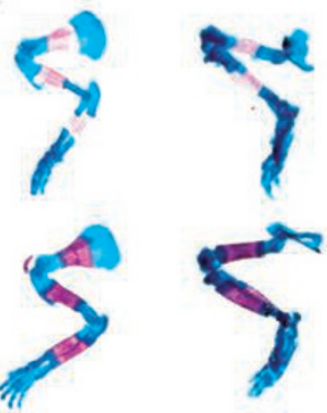

D
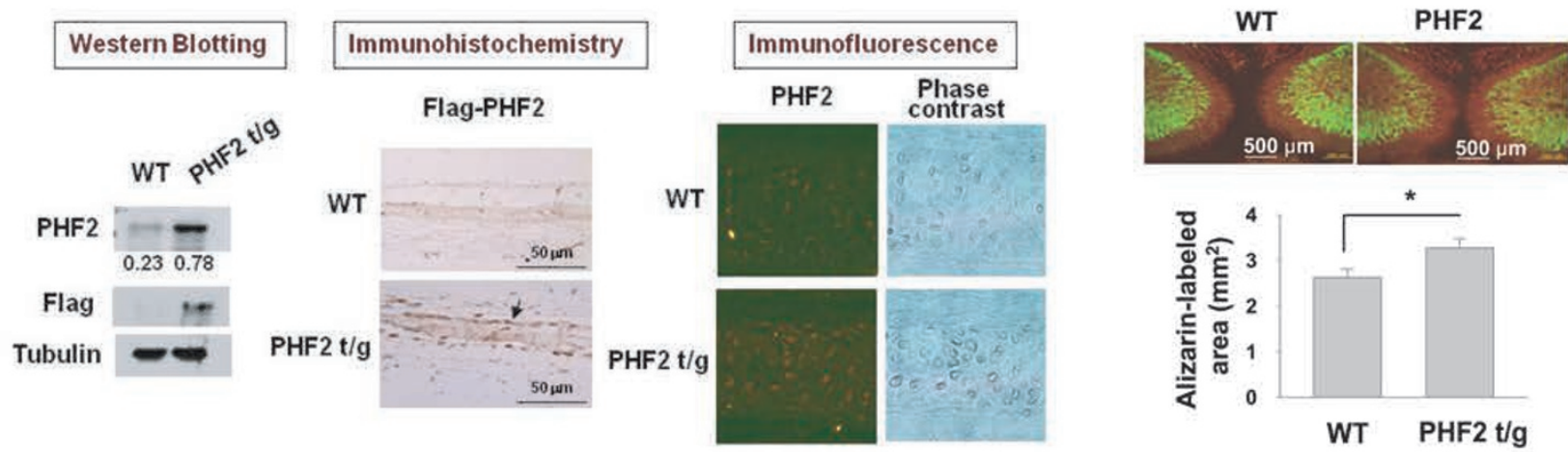

C

E

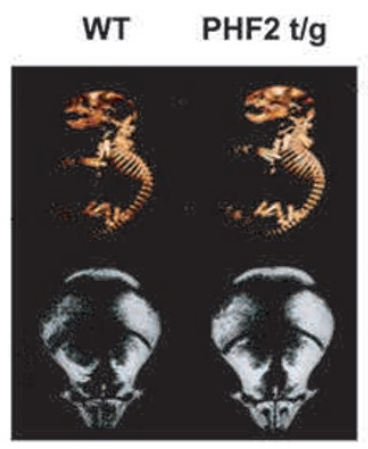

Calvarial bone

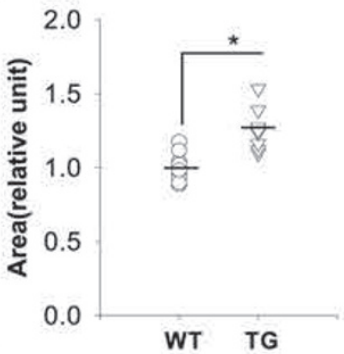

Calvarial bone

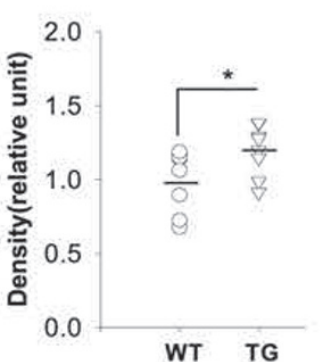

Von Kossa

WT

PHF2 $t / g$

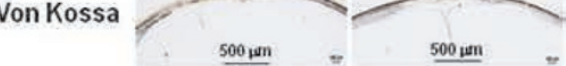

OCN/DAPI
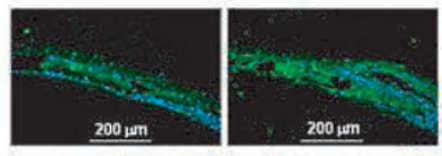

H\&E
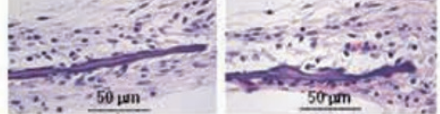

$\mathbf{F}$
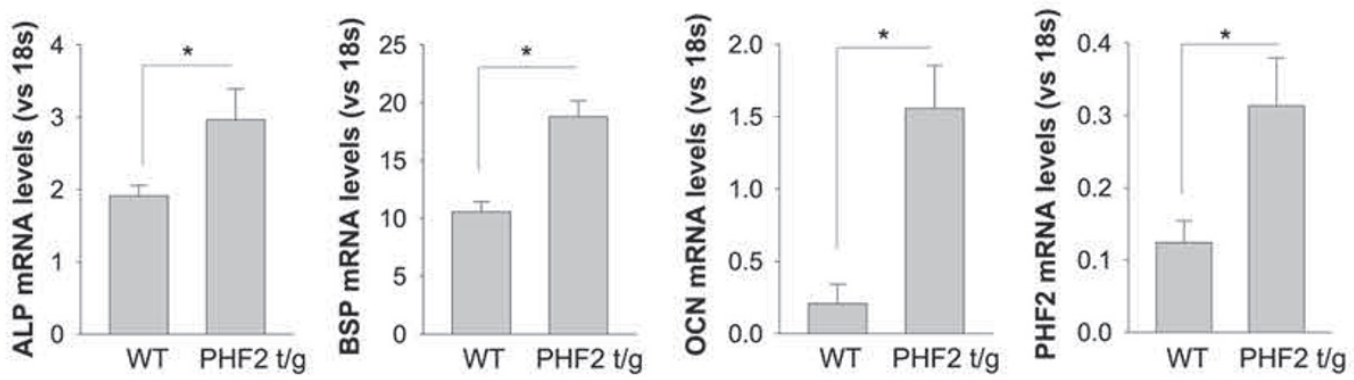
ized in nuclei of wild-type osteoblasts (Figure 2B). To examine whether PHF2 affects the rate of osteoblast differentiation, alkaline phosphatase (ALP) staining (Figure 2C) and its enzymatic activity assay (Figure 2D) were carried out. Both assays revealed an earlier and more evident increase in ALP activity in PHF2-t/g osteoblasts than in wild-type cells (Figure 2C and 2D). The effects of PHF2 on late osteoblast differentiation (maturation) were examined by using Alizarin red staining to assess matrix mineralization and RT-PCR to measure the expression of differentiation markers. PHF2-t/g osteoblasts displayed greater mineralization (Figure 2E) and enhanced expression of ALP, OCN, and BSP (Figure 2F). Consistently, ectopic expression of PHF2 in $\mathrm{C} 2 \mathrm{C} 12$ precursor cells facilitated BMP2-induced ALP activation (Supplementary information, Figure S3). Collectively, these results suggest that PHF2 is a positive regulator of osteoblast differentiation.

\section{PHF2 is essential for osteoblast differentiation}

The involvement of PHF2 in osteoblast differentiation was also examined by knocking down PHF2 in C2C12 cells using shRNA lentivirus. Western blotting confirmed the stable knockdown of PHF2 (Figure 3A). As in primary osteoblasts, BMP2 treatment upregulated the expression of PHF2 and Runx2 in control C2C12 cells (Figure 3A). PHF2 knockdown significantly attenuated ALP activity and matrix mineralization (Figure 3B-3D), as well as mRNA expression levels of osteoblast differentiation markers (ALP, OCN, and BSP; Figure 3E). These results further demonstrate that PHF2 supports osteoblast differentiation.

Bone regeneration in rat calvaria is blocked by $\mathrm{PHF} 2$ knockdown
In addition to bone development, fracture healing also depends on osteoblast differentiation. Therefore, the role of PHF2 in bone regeneration was examined in rats with surgery-generated calvarial defects. Two shRNAs were designed to target rat PHF2, and the efficiency of PHF2 knockdown was verified in rat fibroblasts by western blotting (Figure 4A). Collagen matrices containing BMP2 and shRNA lentiviruses were placed over the calvarial defects. After 8 weeks of recovery, newly formed bones were analyzed by reconstructing $3 \mathrm{D}$ micro-CT images. PHF2 knockdown significantly decreased the regenerated bone volume (Figure 4B and 4C). Histological examination confirmed that tissue formed at defect margins was bone (Figure 4D). These in vivo data suggest that PHF2 is also required for bone regeneration.

\section{PHF2 binds to Runx2 and facilitates its transcriptional activity}

It was recently reported that PHF2 and Runx2 share a similar expression pattern in thymocytes of different developmental stages [17], similar to what we have observed in osteoblasts (Figures 2A and 3A). Therefore, the involvement of PHF2 in Runx2 signaling was examined using osteocalcin promoter (OG2)-luciferase and $6 \times$ OSE-luciferase (a construct containing 6 Runx2-binding sites) reporters. BMP2 treatment activated luciferase expression driven by the $O G 2$ promoter in $\mathrm{C} 2 \mathrm{C} 12$ cells, and PHF2 knockdown negated this effect (Figure 5A). PHF2 knockdown also attenuated the $6 \times$ OSE and OG2 reporter activation induced by ectopic expression of Runx2 (Figure 5B). Conversely, ectopic expression of PHF2 augmented Runx2-stimulated activation of both reporters and a mutant version of PHF2 with lower activity (H249A) [4] did so to a considerably lesser extent (Figure 5C). We next examined the interaction between

Figure 1 PHF2 facilitated bone development in mice. (A) Bones and cartilages of Flag-PHF2-t/g mice and their littermates on postnatal day 1 (P1) were stained with Alizarin Red (red color) and Alcian Blue (blue color), respectively. The left panel shows the representative results for whole skeletons, and the right panel shows calvaria (a), forelimb (b), and hindlimb (c). (B) The expression of transgenic Flag-PHF2 in mouse calvarias on P1 was checked by immunoblotting with anti-PHF2 or anti-Flag antibody (left panel), by immunohistochemistry with an anti-Flag antibody (middle panel), and by immunofluorescence with an anti-PHF2 antibody (right panel). The densities of PHF2 and $\beta$-tubulin blots were quantified using the ImageJ program and the PHF2/tubulin ratios are presented below the PHF2 blot. An arrow indicates a Flag-PHF2-expressing osteoblast in the periosteum. (C) The left panel shows the micro-CT images of bodies (top) and calvarias (bottom) of PHF2-t/g mice and their wild-type littermates on P1. Based on micro-CT images, calvarial bone areas and densities were analyzed using the Image J1.36b software (NIH: Maryland, MD) and plotted in the right panels. Horizontal bars represent mean values, and * denotes $P<0.05$ for the indicated groups. (D) Bone formation in PHF2-t/g mice and their littermates (WT) on P1 was detected by double labeling with Calcein (green) and Alizarin (red). The area of Alizarin-labeled tissue, which indicates bone formed newly for two days before birth, was analyzed using ImageJ and plotted in the bottom panel. Data shown represent the mean $\pm \mathrm{SD}(n=3)$ and * denotes $P<0.05$. (E) In calvarias of mice on P1, mineralization, OCN expression and histology were analyzed by Von Kossa staining, immunofluorescence with anti-OCN antibody and H\&E staining, respectively. (F) The mRNA levels of the osteogenic genes (ALP, BSP, and OCN) and PHF2 in calvarias were analyzed by RT-qPCR and were normalized to 18S RNA levels. Data shown represent the mean $\pm \operatorname{SD}(n=4)$ and * denotes $P<0.05$. 
A

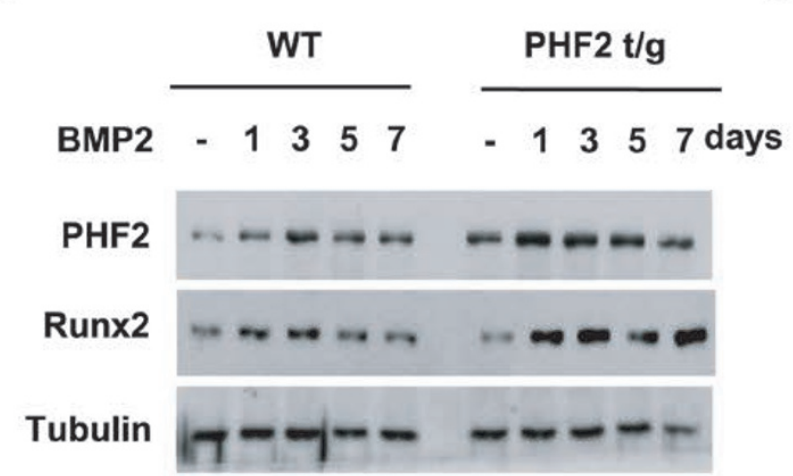

C

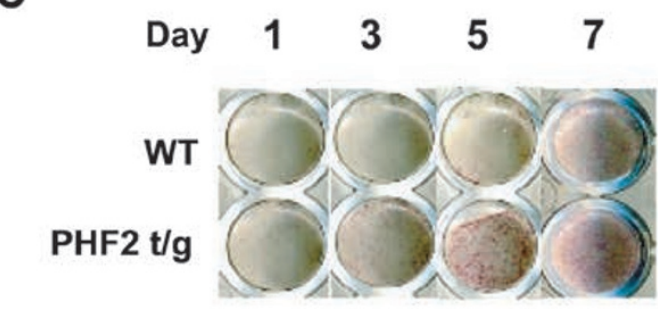

E

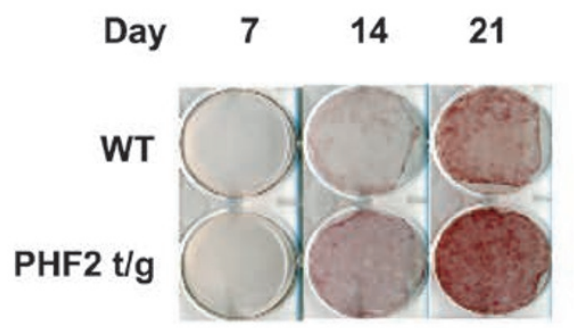

B

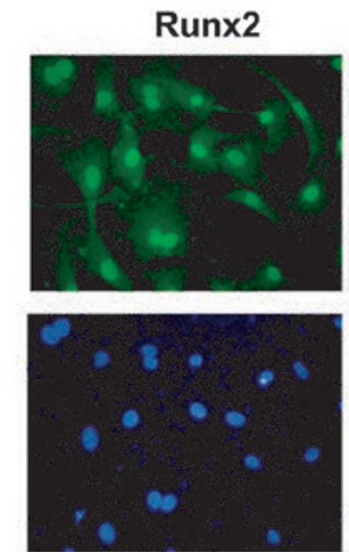

DAPI
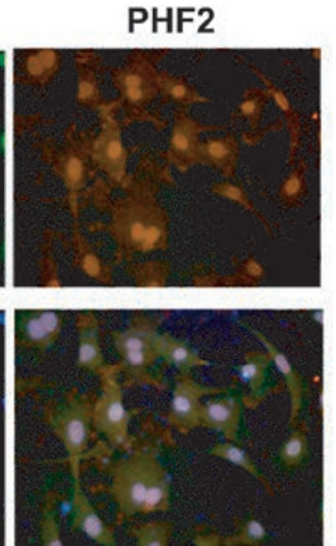

Merge 
A

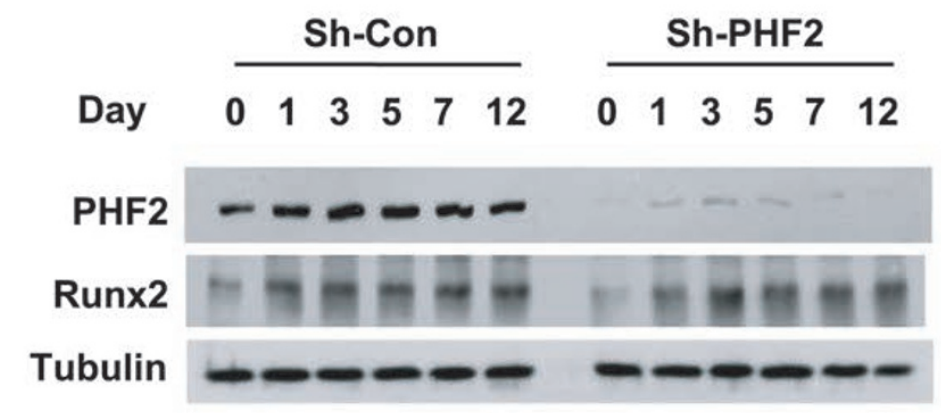

C

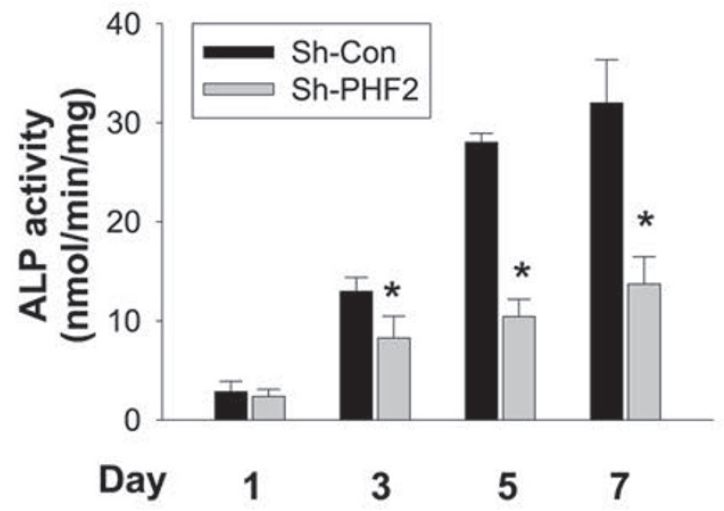

$\mathbf{E}$
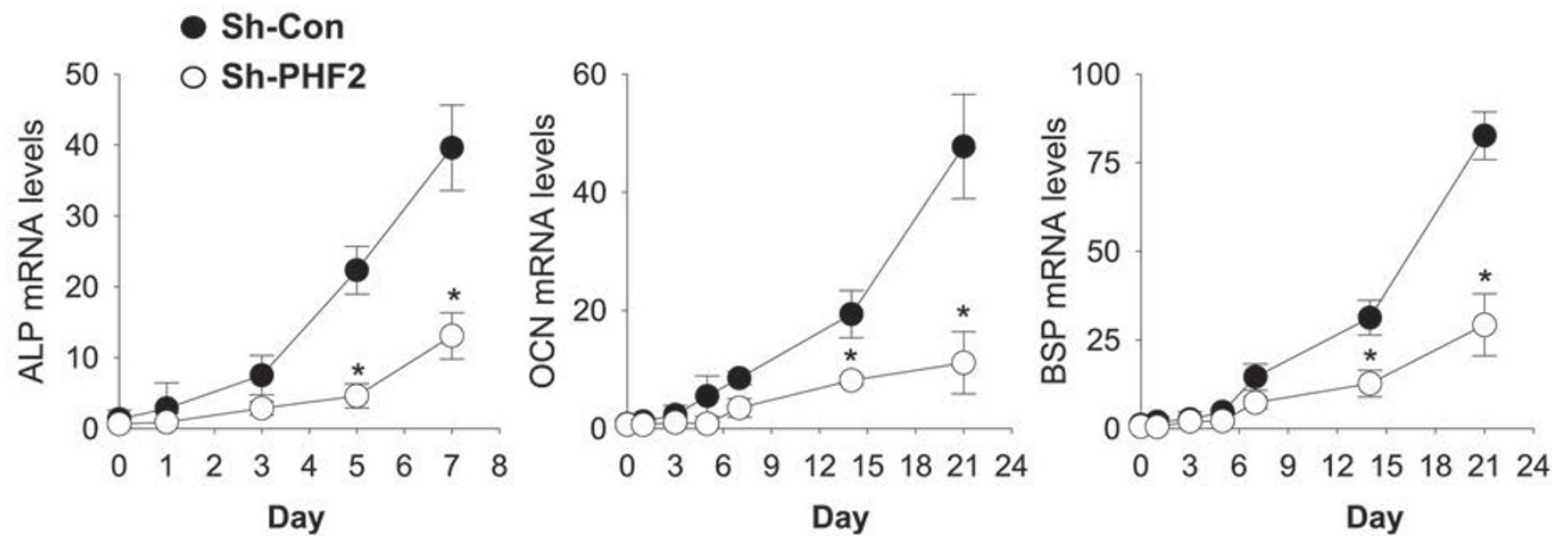

Figure 3 PHF2 was required for osteoblast differentiation. (A) Temporal changes in Runx2 and PHF2 levels in C2C12 cells treated with $100 \mathrm{ng} / \mathrm{ml}$ of BMP2 were analyzed by immunoblotting. (B, C) C2C12 stable cell lines expressing non-targeting (Sh-Con) or PHF2-targeting (Sh-PHF2) shRNA were incubated with BMP2 for the indicated days. C2C12 differentiation toward osteoblasts was monitored by staining ALP (B) and by measuring its activity (C). (D) C2C12 cells under BMP2-induced differentiation were stained with Alizarin. (E) The mRNA expression levels of osteogenic genes were analyzed by RT-qPCR. Each point represents the mean $\pm \operatorname{SD}(n=3)$ of relative values to day 0 and * denotes $P<0.05$ versus the wild-type value. 
Runx2 and PHF2 by co-immunoprecipitation and found that Myc-Runx2 bound to Flag-PHF2 in HEK293T cells (Figure 5D). Co-immunoprecipitation using Runx2 and PHF2 antibodies confirmed the interaction between the endogenous proteins in $\mathrm{C} 2 \mathrm{C} 12$ cells treated with $\mathrm{BMP} 2$ (Figure 5E). To identify the interacting domains, four segments of each protein were generated and immunoprecipitation revealed that the Runt domain (R2) of Runx2 interacts with PHF2 (Figure 5F), and the JmjC domain (P2) of PHF2 interacts with Runx2 (Figure 5G).

\section{PHF 2 enhances DNA binding ability of Runx2 by direct-} ly demethylating Runx2

Given that the Runt domain participates in DNA binding and the JmjC domain catalyzes demethylation, the interaction between these domains was expected to affect the DNA binding ability of Runx2. Therefore, the effect of PHF2 knockdown on the binding of Runx2 to OCN promoter was examined by ChIP. Figure $6 \mathrm{~A}$ shows the locations of Runx2-binding sites within the $O C N$ gene and the regions amplified by real-time PCR in ChIP analysis. PHF2 knockdown efficiency in $\mathrm{C} 2 \mathrm{C} 12$ cells was verified by using western blotting (Figure 6B). As shown above, Runx 2 expression was fully induced within 3 days of BMP2 stimulation (Figures $2 \mathrm{~A}$ and $3 \mathrm{~A}$ ), while $\mathrm{OCN}$ expression started after day 12 in wild-type primary osteoblasts and $\mathrm{C} 2 \mathrm{C} 12$ cells (Figures $2 \mathrm{~F}$ and $3 \mathrm{E}$ ). To understand the time gap between Runx 2 and $\mathrm{OCN}$ expression, we examined the binding of Runx 2 to $O C N$ promoter on days 3 and 12 after BMP2 treatment. As expected, ChIP analyses showed that the binding of Runx 2 to both target elements in $O C N$ promoter was stimulated by BMP2 at both time points. Interestingly, Runx 2 binding to $O C N$ promoter significantly increased on day 12 compared with that on day 3 (Figure 6C), suggesting that it takes time for the chromatin context of the Runx2-binding site in the $O C N$ gene to become accessible. PHF2 knockdown significantly reduced Runx 2 binding at both time points (Figure 6C). Interestingly, PHF2 was also recruited to Runx2 target elements under BMP2 stimulation, which was also further increased on day 12 (Figure 6D). Because PHF2 is known to demethylate histone H3, we tested whether PHF2 regulates Runx2-driven transcription by modifying the methylation status of $\mathrm{H} 3$. As shown in Figure 6E, PHF2 knockdown increases dimethylation levels at $\mathrm{H} 3 \mathrm{~K} 9$ and $\mathrm{H} 3 \mathrm{~K} 27$. Given that PHF2 demethylates $\mathrm{H} 3 \mathrm{~K} 9 \mathrm{me} 2$ [1], we examined the levels of $\mathrm{H} 3 \mathrm{~K} 9 \mathrm{me} 2$ in stable-C2C12 cells expressing control or PHF2 shRNA in the presence or absence of BMP2 treatment. H3K9me2 levels on Runx2 target segments were enhanced in BMP2-treated control cells. Unexpectedly, H3K9me2 levels under BMP2 stimulation were barely changed by PHF2 knockdown (Figure 6F). Therefore, the decreased DNA binding affinity of Runx2 to its target segments upon PHF2 knockdown is unlikely due to changes in local $\mathrm{H} 3 \mathrm{~K} 9 \mathrm{me} 2$ levels. These results encouraged us to investigate whether PHF2 directly demethylates Runx2. When PHF2 was knocked down, lysyl methylation level of Runx2 increased sharply in both $\mathrm{C} 2 \mathrm{C} 12$ cells and primary osteoblasts (Figure 6G). To identify which residue in Runx2 was methylated, Runt peptide (R2 in Figure 5F) purified from BMP2-stimulated C2C12 cells was examined by liquid chromatography-mass spectrometry (LC-MS) and peptide methylation was confirmed using an anti-methylated lysine antibody (Supplementary information, Figure S4A). Based on mass spectra, the Runt peptide was not methylated in the presence of PHF2 (Supplementary information, Figure S4B), but was dimethylated at K245 (in mouse Runx2) upon PHF2 knockdown (Supplementary information, Figure S4C). As a BLAST search revealed that the dimethylated lysine residue in Runx 2 is well conserved among vertebrate species (Figure $6 \mathrm{H}$ ), we examined whether K245 was the target of PHF2 by examining the methylation status of a mutated Runt peptide K245A in $\mathrm{C} 2 \mathrm{C} 12$ cells. The mutated peptide was methylated less than the wild-type (Figure 6I) but methylation was not eliminated, suggesting the presence of another methylation site in Runx2. Surprisingly, the K245A mutation attenuated PHF2-induced activation of Runx 2 in both $6 \times \mathrm{OSE}$ and OG2 reporter assays (Supplementary information, Figure S5). Theoretically, as PHF2 facilitates the binding of Runx2 to target DNA and Runx2-K245A cannot be methylated, this Runx 2 mutant is expected to be constitutively active, which is not consistent with the results of the reporter assays (Supplementary information, Figure S5). As the structure of alanine is quite different from that of lysine, the K-to-A mutation seems to decrease the functionality of Runx2. Therefore, we next substituted K245 with arginine, which should minimize the conformational change of Runx2, as arginine is the most similar to lysine among other amino acids. Indeed, compared with wildtype Runx2, Runx2-K245R showed a higher transcriptional activity that was no longer regulated by PHF2 (Figure 6J). Also, Runx2-K245R bound more strongly to $O C N$ promoter than wild type (Figure $6 \mathrm{~K}$ ). These results indicate that PHF2-mediated demethylation of K245 is a critical step in regulating Runx2-driven transcription.

\section{SUV39H1 methylates Runx2}

Because SET domain-containing histone lysine methyltransferase (SET-HKMT) enzymes are known to target both histones and non-histone proteins [18-20], whether SET-HKMT family members bind to and methylate 
A

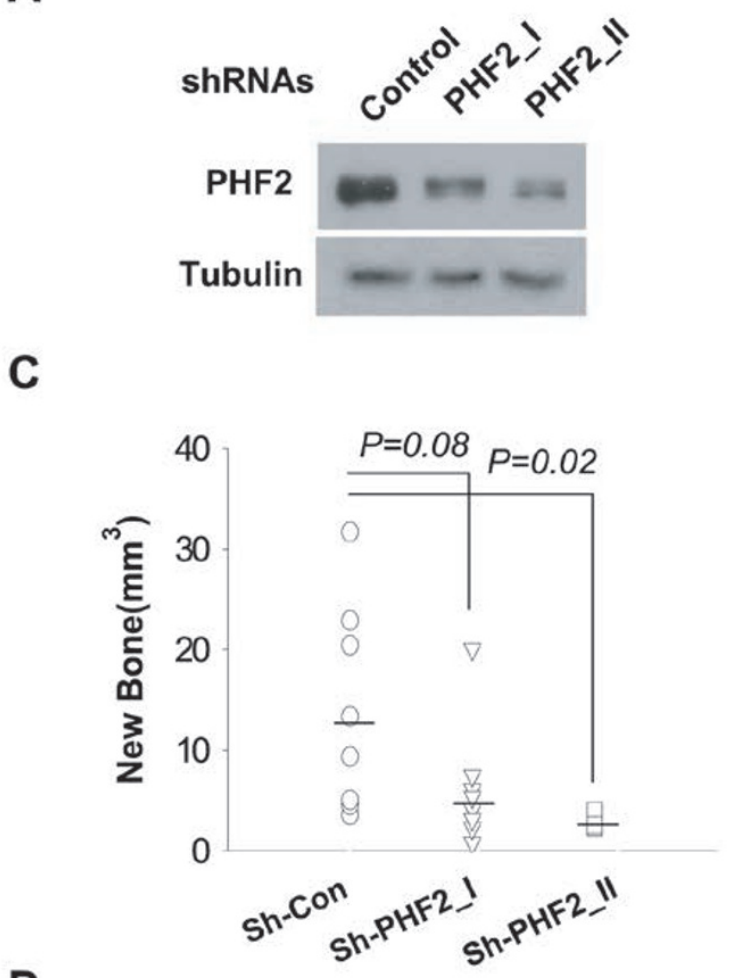

B

\begin{tabular}{lll}
\multicolumn{2}{c}{ shRNAs } \\
\hline Control PHF2_I & PHF2_II
\end{tabular}
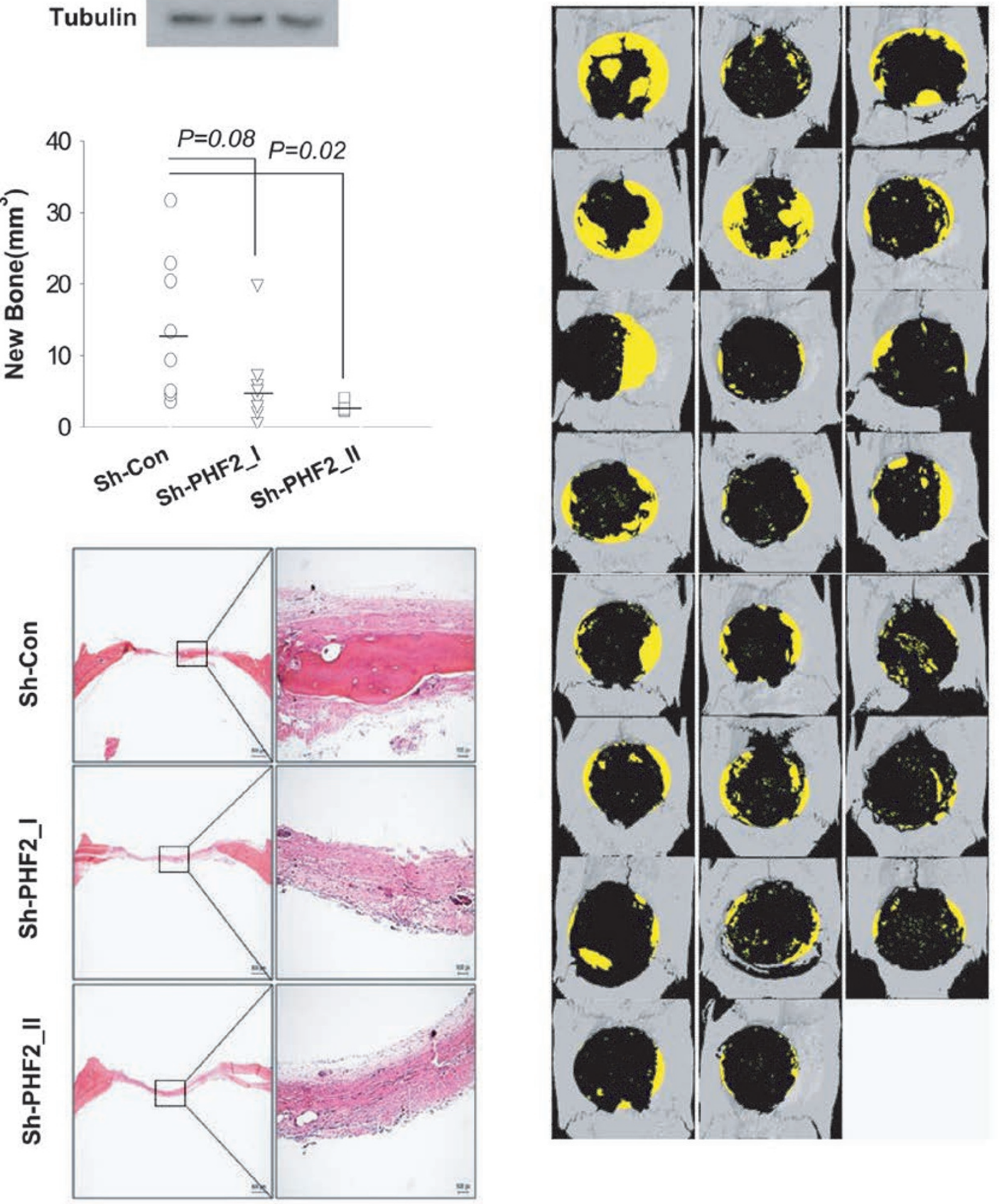

Figure 4 PHF2 was essential for bone regeneration in calvaria of adult rats. A part of rat calvarial bone was removed, and the defect was covered for 8 weeks with a collagen sponge containing $2 \mathrm{mg}$ of BMP2 and $5 \times 10^{9}$ of shRNA lentivirus. (A) The efficiencies of two shRNAs targeting PHF2 were verified by infecting rat fibroblasts with shRNAs, followed by immublotting PHF2. (B) A 3D micro-CT analysis was conducted to visualize and quantify bone regeneration. Yellow zones represent the new bone growing from defect margins. (C) The volume of the new bone was evaluated by 3D micro-CT images and plotted as individual points. Horizontal bars represent mean values (Sh-Con, $n=8$; Sh-PHF2_I, $n=8$; Sh-PHF2_II, $n=7$ ). (D) Calvarial sections were stained with H\&E. Scale bars, left column $800 \mu \mathrm{m}$, and right column $100 \mu \mathrm{m}$. 
Runx2 was addressed. G9a and SUV39H1 were examined first as candidate Runx2 methyltransferases. Immunoprecipitation showed that Myc-Runx2 co-precipitated with Flag-SUV39H1, but not with Flag-G9a (Figure 7A). As a positive control, we also observed the presence of Flag-CBF $\beta$ in Myc-Runx2 immunoprecipitates (Figure
7A). The interaction between endogenous Runx2 and SUV39H1 was established in $\mathrm{C} 2 \mathrm{C} 12$ cells. Interestingly, the Runx2-SUV39H1 interaction was weakened under BMP2 stimulation (Figure 7B). To determine whether SUV39H1 and PHF2 directly methylates and demethylates Runx2, respectively, we performed methylation
A

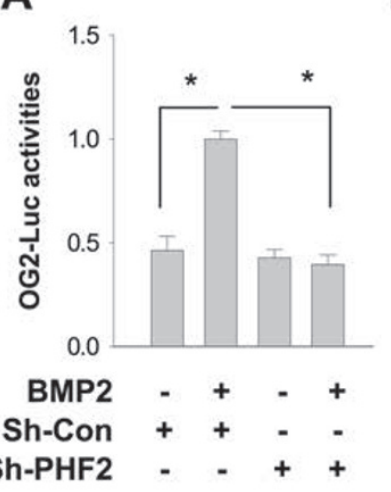

D

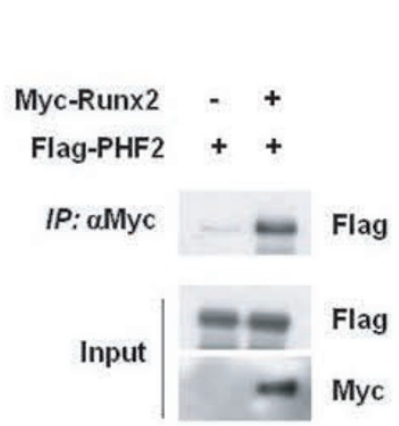

B

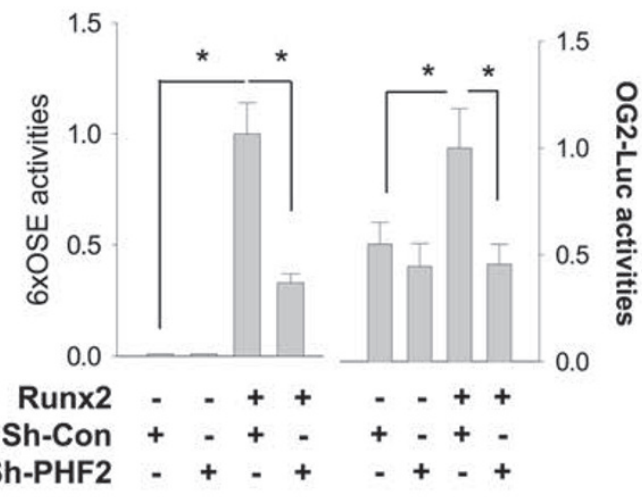

\section{E}

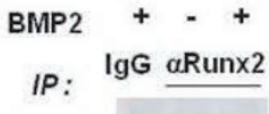

Bound

PHF2

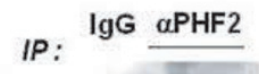

Bound

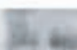

Input

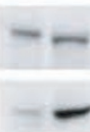

PHF2

Runx2
C
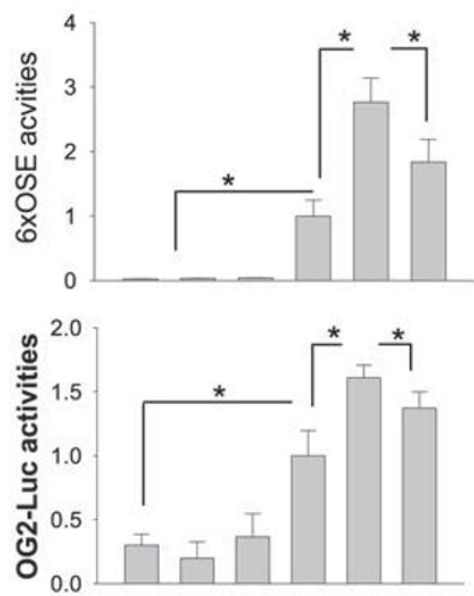

Myc-Runx2 - - - + + +

Vector + - - + - -

Flag-PHF2 - + - - + +

Flag-PHF2 - $-+\quad-\quad+$

(H249A)

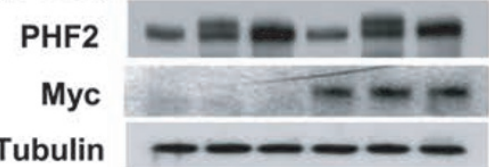

$\mathbf{F}$
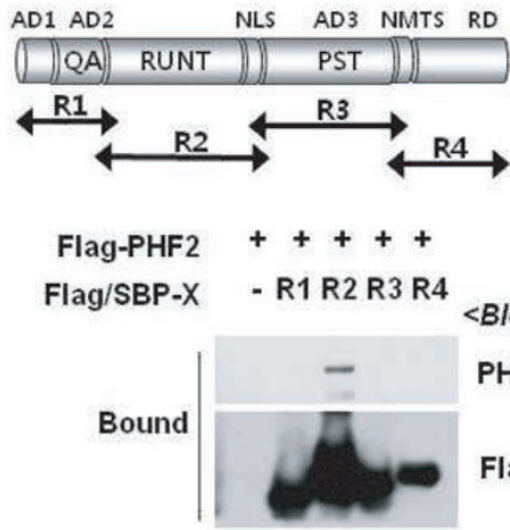

$<$ Blot $>$

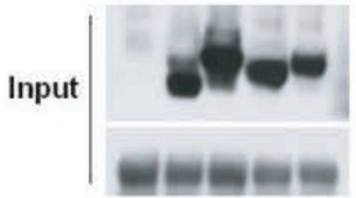

PHF2

Flag

Flag

PHF2

G
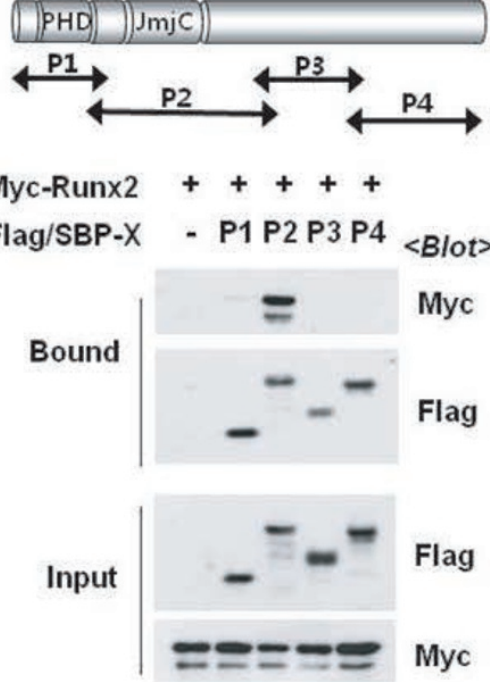
assays using a recombinant Runt peptide in vitro. Incubation with SUV39H1 and the methyl donor SAM led to R2 peptide methylation, which was reversed by PHF2 (Figure 7C). SUV39H1-mediated methylation was then confirmed at the cellular level; expression of SUV39H1 induced lysyl methylation of the Runt peptide, which was reduced by co-expression of PHF2, while the methylation level of the peptide with K245A mutation can barely be enhanced upon SUV39H1 overexpression (Figure 7D). We next tested whether K245R mutation affects the recruitment of PHF2 or SUV39H1 by Runx2, and found that this mutation enhances Runx2 binding to SUV39H1 but not to PHF2 (Supplementary information, Figure S6), suggesting that SUV39H1 may have higher affinity for the constantly demethylated substrate Runx2-K245R than for the methylated Runx 2 . These results suggest that the lysyl methylation of Runx 2 is counterbalanced by SUV39H1 and PHF2.

\section{SUV39H1 delays osteoblast differentiation by inhibiting Runx2-driven transcription}

To understand the functional consequence of SUV39H1-induced methylation of Runx2, we knocked down SUV39H1 in C2C12 cells, and treated the cells with BMP2 to induce osteoblast differentiation. Upon SUV39H1 knockdown, ALP activity, matrix mineralization (Figure 8A) and the expression levels of ALP, OCN, and BSP were increased (Figure 8B) and Runx2 activity, as assessed by OG2 reporter expression, was enhanced (Figure 8C). Moreover, SUV39H1 expression inhibited Runx2 activity, and PHF2 co-expression recovered it (Figure 8D). More importantly, Runx2K245R was fully activated regardless of SUV39H1 or PHF2 expression (Figure 8D). ChIP analyses showed that binding of Runx2 to $O C N$ promoter was negatively regulated by SUV39H1 (Figure 8E and 8F). Interestingly, SUV39H1 was also recruited to $O C N$ promoter (Supplementary information, Figure S7A and S7B), and was found to induce dimethylation of H3K9 under BMP2 stimulation (Supplementary information, Figure S7C and S7D). These results suggest that SUV39H1 controls Runx2-driven transcription both by inhibiting Runx2 binding to DNA and by methylating H3K9 around Runx2 target elements. Taken together, our findings suggest that SUV39H1 and PHF2 reciprocally regulate the lysyl methylation of Runx 2 and thus act to balance osteoblast differentiation, as summarized in Figure 8G.

\section{Discussion}

In the current study, we report for the first time that the lysine demethylase PHF2 plays an essential role in bone formation. In comparison to their wild-type littermates, the calvarial and limb bones of PHF2 transgenic mice developed earlier. In addition, PHF2 knockdown slowed-down bone regeneration in injured rat calvaria. In primary osteoblasts and $\mathrm{C} 2 \mathrm{C} 12$ cells, PHF2 expression was induced along with Runx 2 expression during differentiation, and osteoblast differentiation progressed in a PHF2-dependent manner. Mechanistically, the DNA-binding ability and transcriptional activity of Runx2 were inhibited by SUV39H1 but were recovered by PHF2. SUV39H1 and PHF2 methylates and demethylates Runx2, respectively. Although SUV39H1 and PHF2 are generally known as epigenetic regulators that modify chromatin structure, they appear to act as post-translational regulators of Runx2 in differentiating osteoblasts. Functionally, PHF2 and SUV39H1 counterbalanced osteoblast differentiation. These results provide new evidence that PHF2 and SUV39H1 are potential

Figure 5 PHF2 interacted with Runx2 to activate Runx2. (A) C2C12 stable cell lines expressing control (Sh-Con) or PHF2-targeting (Sh-PHF2) shRNA were transfected with OG2 promoter-luciferase reporter and $\beta$-gal plasmids and treated with $50 \mathrm{ng} / \mathrm{ml}$ of BMP2 for $24 \mathrm{~h}$. Bars represent the mean $\pm \mathrm{SD}(n=4)$ for relative luciferase activity. * denotes $P<0.05$ for the indicated groups. (B) $\mathrm{C} 2 \mathrm{C} 12$ stable cell lines were co-transfected with Myc-Runx2, luciferase reporter, and $\beta$-gal plasmids, and incubated for $48 \mathrm{~h}$. Bars represent the mean \pm SD $(n=4)$. (C) C2C12 cells were co-transfected with Myc-Runx2, Flag vector, Flag-PHF2, Flag-PHF2 (H249A), $6 \times$ OSE-luciferase, OG2 promoter-luciferase, and $\beta$-gal plasmids in the indicated combinations. Results (luciferase/ $\beta$-gal) are presented as the relative values to the BMP2/Sh-Con group. Bars represent the mean \pm SD $(n=4)$. (D) HEK293T cells were co-transfected with Myc-Runx2 and Flag-PHF2 plasmids. Cell extracts were immunoprecipitated with an anti-Myc antibody, and coprecipitated Flag-PHF2 was identified using anti-Flag. (E) After C2C12 cells were stimulated with BMP2 for $24 \mathrm{~h}$, endogenous Runx2 or PHF2 was precipitated with anti-Runx2 or anti-PHF2, and coprecipitated proteins were immunoblotted. (F) The top panel shows a schematic diagram of Runx2. HEK293T cells were co-transfected with Flag-PHF2 and one of four plasmids for Flag/SBP-Runx2 fragments. Runx2 fragments were pulled down using streptavidin beads, and the precipitated proteins were immunoblotted. AD1-3, transactivation domain 1-3; NLS, nucleus localization signal; NMTS, nuclear matrix-targeting signal; RD, repression domain; QA, polyglutamine and polyalanine domain; PST, proline/serine/threonine-rich domain. (G) The top panel shows a schematic diagram of PHF2. HEK293T cells, which had been co-transfected with Myc-Runx2 and one of four F/S-PHF2 fragments, were subjected to streptavidin pulldown, and the precipitated proteins were immunoblotted. 
A

Run $\times 2$ Binding Sites

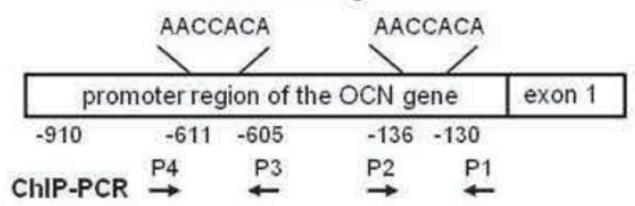

D

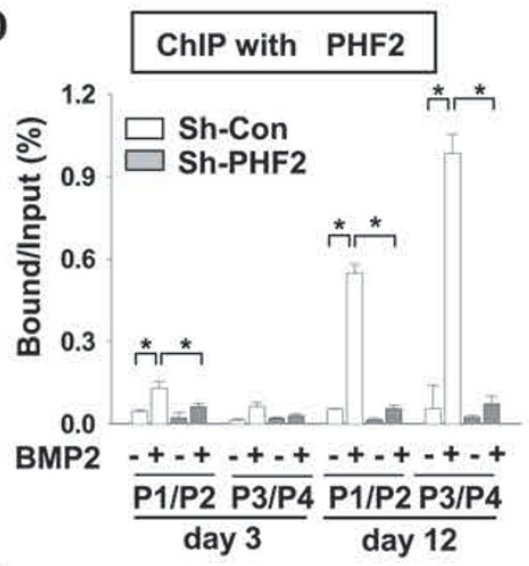

F

\section{ChIP with H3K9me2}

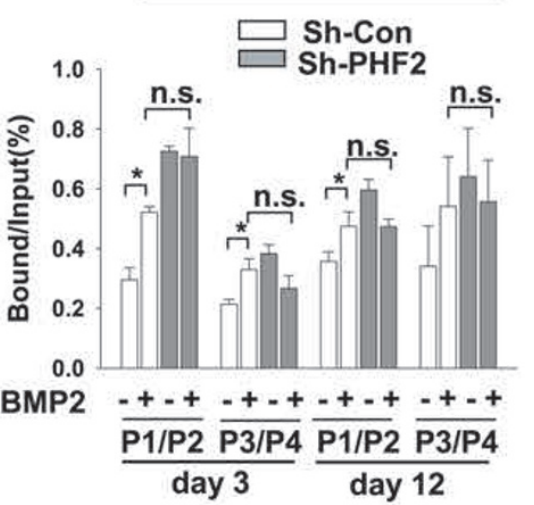

I

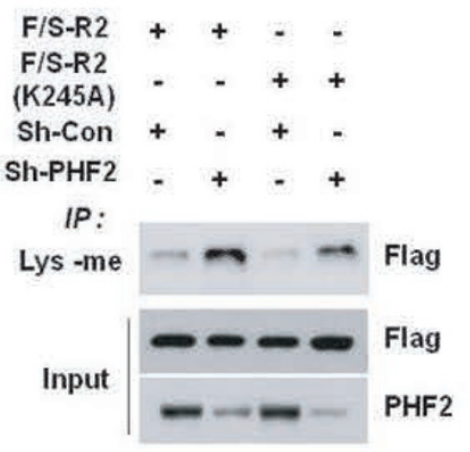

B

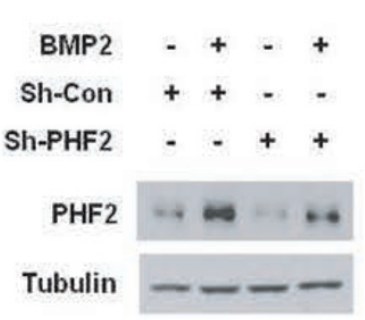

E

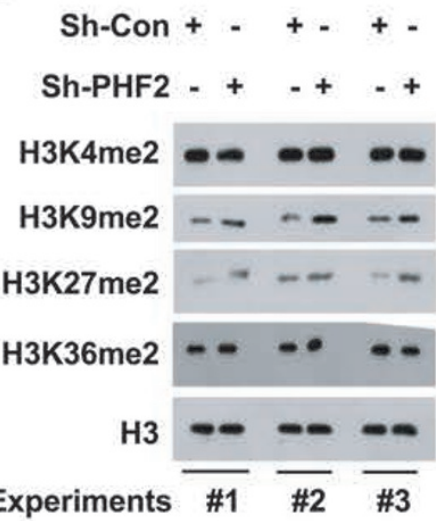

G

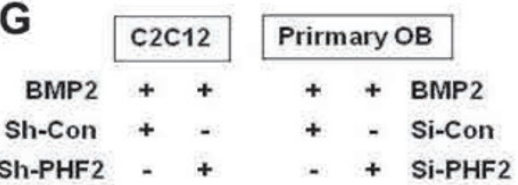

IP :

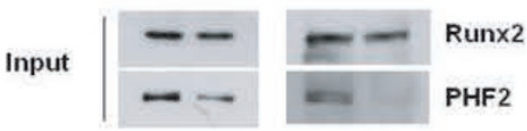

$$
\text { J }
$$
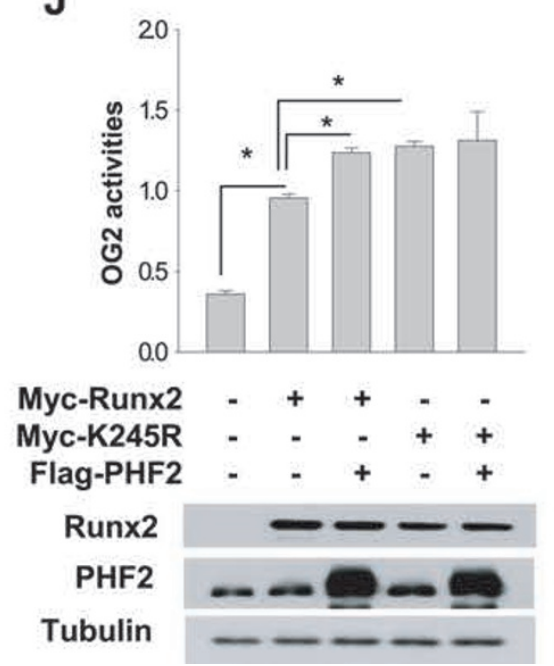

C
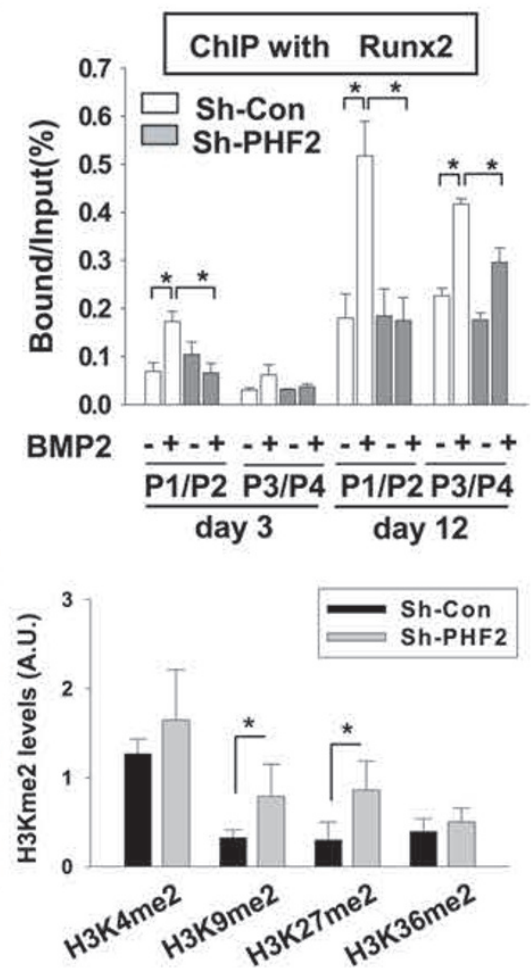

H

[Conserved Runx2 domain ]

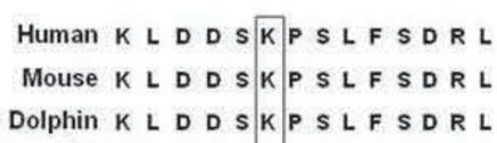

Dolphin KLDDSKPSLFSDRL Bat $K L D D S K P S L F S D R L$

K

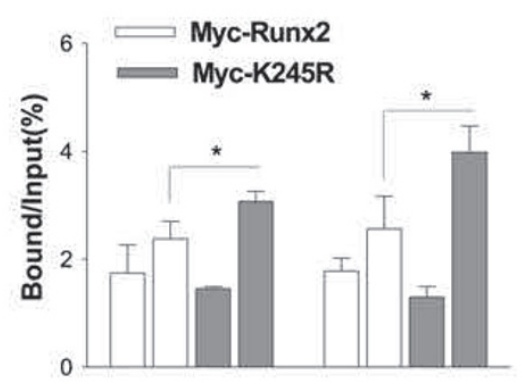

BMP2 $\frac{-+-+}{\text { Myc }} \frac{-+-+}{\text { P1/P2 }} \frac{\text { Myc }}{\text { P3/P4 }}$ 
therapeutic targets in diseases associated with impaired bone development or delayed fracture healings.

Post-translational modifications (PTMs) change the chemical nature and structure of amino acids and lead to diversity in the localization, stability, and function of proteins. Runx 2 activity has been known to be regulated by three PTMs, phosphorylation, acetylation, and ubiquitination [21]. Phosphorylation of human Runx2 variant 1 (NM_001024630) at S28, S247, and S340 induced by FGF-2 and parathyroid hormone enhances its transcriptional activity $[22,23]$. In contrast, phosphorylation at $\mathrm{S} 118, \mathrm{~S} 369, \mathrm{~S} 373, \mathrm{~S} 377$, or S465 has been demonstrated to inhibit Runx2-driven transcription [9, 24]. In addition, a previous research has demonstrated that the p300 coactivator acetylates and activates Runx2 under BMP2 stimulation and that mutations of K233, K238, $\mathrm{K} 358$, and K359 abolished p300-dependent acetylation, suggesting that all or some of these lysine residues are acetylated [25]. Ubiquitination of Runx 2 by the ubiquitin E3 ligase Smurf1 induces degradation of Runx2 [12]. To the authors' knowledge, no study has demonstrated methylation of Runx 2 per se. The present study is the first to show that mouse Runx2 is methylated at K245 (K238 in human Runx2) and that this methylation is counterbalanced by SUV39H1 and PHF2.

Our results suggest that lysyl methylation negatively controls the transcriptional activity of Runx2. Methylation occurs in the Runt domain, which is responsible for the binding of Runx2 to $O C N$ promoter, and the DNA-binding ability of Runx 2 was attenuated by SUV39H1 (Figure 8E) but augmented by PHF2 (Figure $6 \mathrm{C}$ ). In two reporter analyses, Runx2-driven transcription was inhibited by SUV39H1 (Figure 8D) but enhanced by PHF2 (Figure 5C). Finally, osteoblast differentiation and bone formation were facilitated when Runx 2 was demethylated by PHF2 (Figures 1-4). Though the structural basis for the effect of dimethylation on binding of Runx2 to DNA was not directly investigated, a likely mechanism is that methylation interferes with the ability of Runx2 to interact with chromatin.

SUV39H1 and G9a are SET domain lysine methyltransferases that commonly catalyze mono-, di-, and tri-methylation of H3K9. In addition, they can transfer the methyl moiety to non-histone substrates. For example, G9a has been found to methylate p53, WIZ, CDYL1, ACINUS, and Reptin [19, 20, 26, 27]. Similarly, Chakraborty et al. [28] have reported that SUV39H1 interacts with the N-terminus of Runx1 (alternatively called AML1), and suggested that SUV39H1 inhibits the transcriptional activity of Runx 1 by blocking its DNA-binding ability. However, it is still controversial whether SUV39H1 directly methylates Runx1 as the methylation level of Runx1 was not significantly augmented by SUV39H1 overexpression [28]. In terms of protein sequences, human Runx1 (NM_001754) and Runx 2 are $56 \%$ identical with $91 \%$ identity within the Runt domains. Here we found that Runx2 was methylated and inactivated by SUV39H1. SUV39H1 has been reported to co-immunoprecipitate with Runx1 and Runx3, but not with Runx2 upon ectopic expression in COS7 cells [29]. In contrast, a recent study [30] has demonstrated that SUV39H1 and Runx2 are co-precipitated in lung cancer cells and recruited together to the $B M P-3 B$ promoter (a Runx2 target). Our study showed that SUV39H1 binds to Runx2 and regulates Runx2 activity via lysyl methylation. These inconsistencies suggest that the interaction between SUV39H1 and Runx2 may depend on the cellular context. Our findings indicate that differentiating osteoblast permits the interaction.

The methylation state and regulation mechanism of

Figure 6 PHF2 demethylated Runx2 and promoted the binding of Runx2 to DNA. (A) A schematic representation of OCN promoter and segments amplified by PCR. (B) C2C12 stable cell lines were incubated with BMP2 for 12 days, and PHF2 was immunoblotted. (C, D) ChIP for the binding of Runx2 or PHF2 to OCN promoter. C2C12 stable cell lines were incubated with BMP2 for 12 days. Chromatin was cross-linked and immunoprecipitated using anti-Runx2 (C) or anti-PHF2 (D). Precipitated DNAs were amplified and quantified by real-time PCR. The results (mean $\pm \mathrm{SD}, n=3$ or more) are expressed as percentages of the input level. (E) The levels of dimethylated H3K4, H3K9, H3K27, H3K36, and H3 were analyzed by immunoblotting in $\mathrm{C} 2 \mathrm{C} 12$ stable cell lines. (F) ChIP was performed six times with anti-H3K9me2 antibody in C2C12 stable cell lines. * and n.s. denote $P<0.05$ and non-significant, respectively. (G) C2C12 stable cell lines or primary osteoblasts, which had been transfected with control or PHF2 siRNA, were treated with BMP2 for $24 \mathrm{~h}$. Lysyl-methylated Runx2 was precipitated using anti-methylated lysine (Lys-me), and precipitated Runx2 was immunoblotted. (H) Comparisons of Runx2 sequences among human, mouse, dolphin, and bat. (I) C2C12 stable cell lines transfected with the F/S-R2 or F/S-R2 (K245A) plasmid were subjected to immunoprecipitation with anti-Lys-me, and the precipitated F/S-R2 peptides were immunoblotted. (J) C2C12 cells were co-transfected with Myc-Runx2 (or its K245R mutant), OG2 promoter-luciferase reporter, Flag-PHF2, and $\beta$-gal plasmids. The results indicate the mean \pm SD $(n=4)$. (K) ChIP was performed to identify the binding of Myc-Runx2 and MycRunx2 (K245R) to OCN promoter. Transfected C2C12 cells were incubated with BMP2 for $48 \mathrm{~h}$. ChIP was done with anti-Myc, and the precipitated DNA fragments were analyzed by real-time PCR. The results (mean \pm SD, $n=3$ ) are expressed as percentages of the input level. * denotes $P<0.05$. 
A

$\begin{array}{cl}\text { Myc-Runx2 } & ++++ \\ \text { Flag-SUV39H1 } & -+-- \\ \text { Flag-G9a } & --+- \\ \text { Flag-CBF } & ---+\end{array}$

IP: Flag

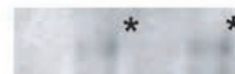

Input
B

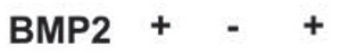

\section{IP : IgG aSUV39H1}

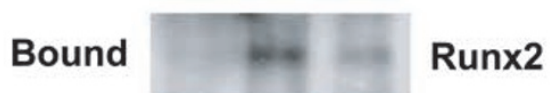

IB: Flag

Input

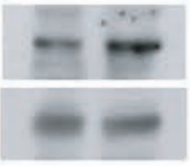

Runx2

SUV39H1

IB: Myc

C

Flag
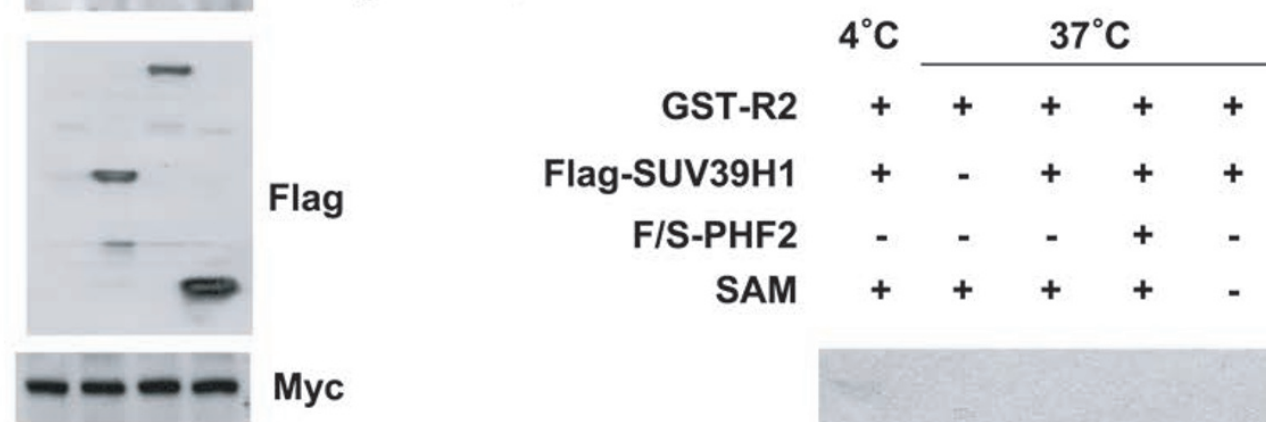

D

F/S-R2

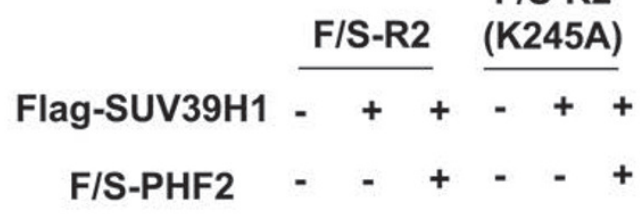

IP:

Lys-me

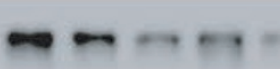

<blot>

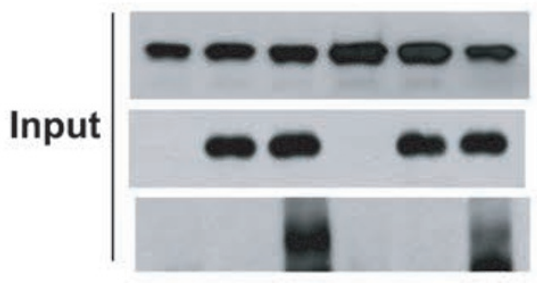

F/S-R2

F/S-R2

SUV39H1

me-Lys

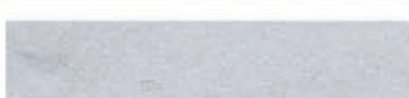

\section{F/S-PHF2}

Figure 7 SUV39H1 methylates Runx2 at Lys245. (A) HEK293T cells were co-transfected with Myc-Runx2 and Flag-SUV39H1, Flag-G9a or Flag-CBF $\beta$ plasmids. Cells were subjected to immunoprecipitation with anti-Myc or anti-Flag, and coprecipitated proteins were immunoblotted. Specific signals for precipitated proteins are asterisked. (B) The differentiation of C2C12 cells was induced by BMP2 treatment for $24 \mathrm{~h}$. Cell lysates were subjected to immunoprecipitation with anti-SUV39H1, and the precipitated proteins were immunoblotted with anti-Runx2. (C) Recombinant GST-R2 peptide was incubated with S-adenosylmethionin (SAM), Flag-SUV39H1 and/or Flag-SBP-PHF2 (F/S-PHF2) peptide at $4{ }^{\circ} \mathrm{C}$ or $37{ }^{\circ} \mathrm{C}$ for 60 min. The methylation of GST-R2 peptide was detected using anti-methylated lysine antibody (upper panel), and proteins in the reaction mixtures were immunoblotted (bottom panel). (D) HEK293T cells were co-transfected as indicated and the cell lysates were subjected to immunoprecipitation with an anti-Lys-me antibody. Precipitated F/S-R2 or K245A mutants were identified using anti-Flag. 
A

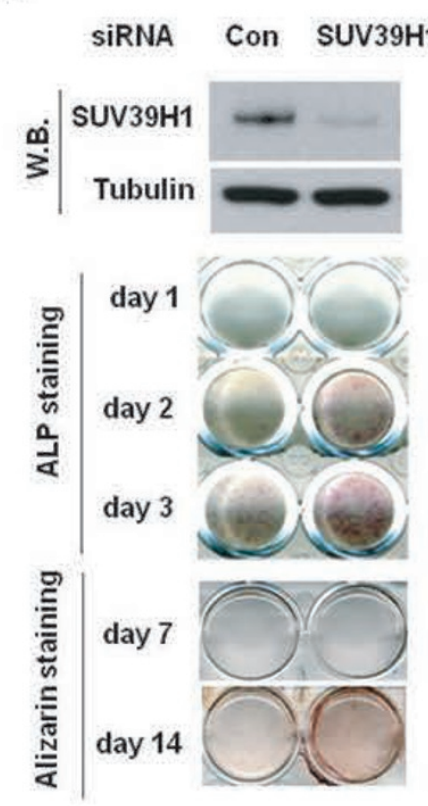

E

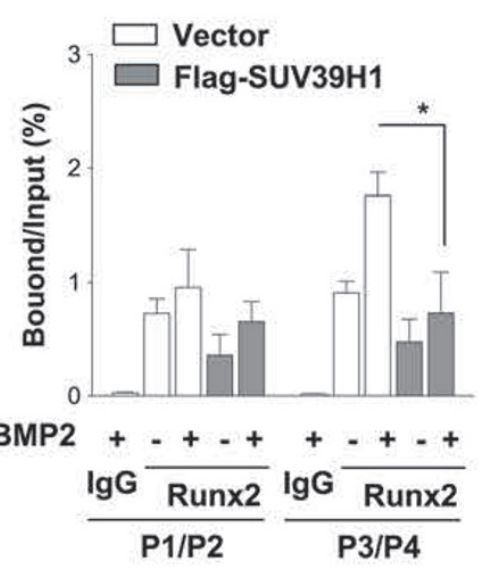

$\mathbf{F}$

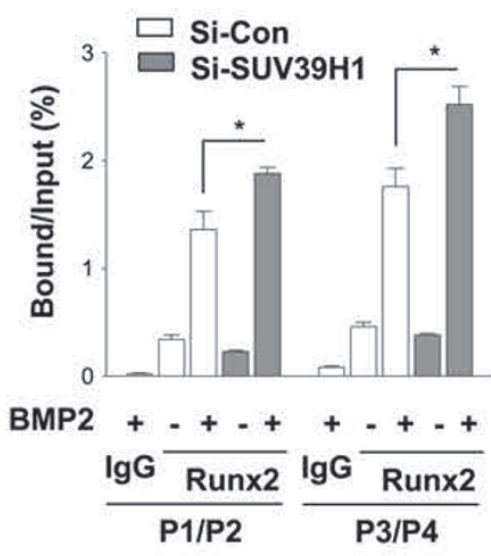

B

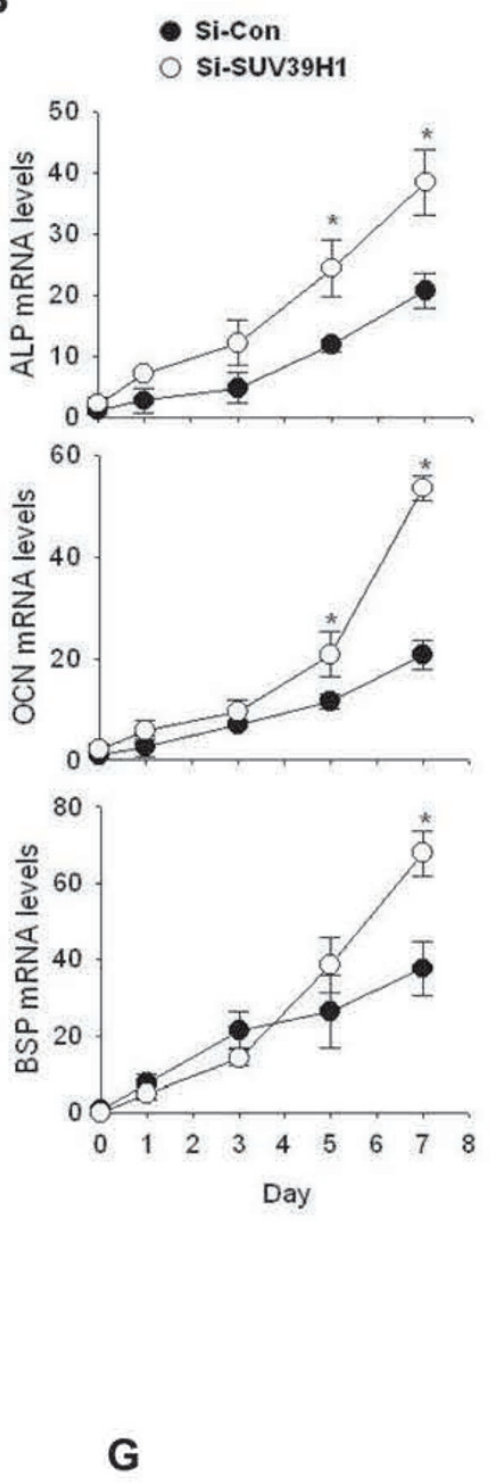

C

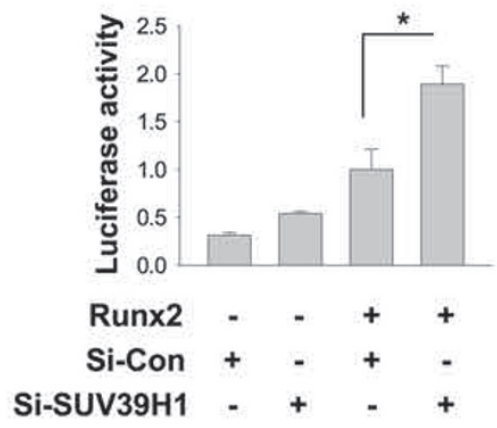

D

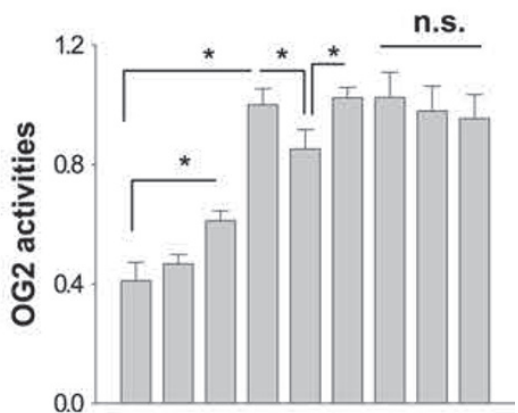

Runx2 - - - + + + - -

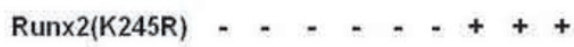

Flag + - - + - + + -

Flag-SUV39H1 - + - + + + +

Flag-PHF2 - - + - + - +

PHF2 $-0-0-0$

Runx2 $-\cdots= \pm= \pm=$

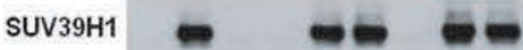

Tubulin $-2-\infty-\infty-\infty$
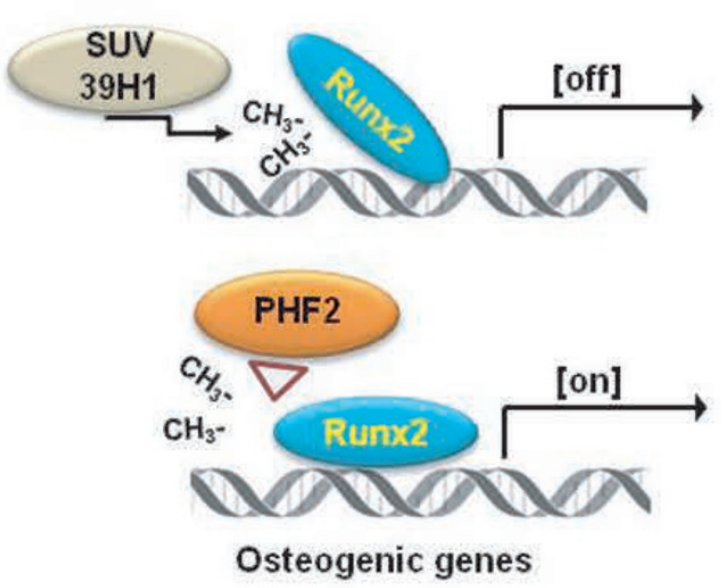
H3K9 and Runx2-K245 are very similar. Both lysine residues can be dimethylated by SUV39H1 and demethylated by PHF2. In addition, there is little difference in the functional consequences of lysine methylation. $\mathrm{H} 3 \mathrm{~K} 9 \mathrm{me} 2$ is known as a marker of transcriptional repression, and dimethylation of Runx2-K245 has been found to be related to repression of Runx2 activity. Thus, SUV39H1 and PHF2 may participate in Runx2-driven gene expression through chromatin remodeling as well as post-translational modification of Runx2. Given these relationships, we examined methylation status of $\mathrm{H} 3 \mathrm{~K} 9$ in the Runx2-binding region of $O C N$ promoter, but observed little change upon PHF2 knockdown (Figure 6F). This suggests that at least for OCN transcription, Runx2 activation by PHF2 is likely more related to Runx 2 demethylation than $\mathrm{H} 3 \mathrm{~K} 9$ demethylation. In contrast, SUV39H1 may repress Runx2-driven transcription via dimethylation of both Runx2-K245 and H3K9 (Supplementary information, Figure S7), which raises a question: which histone code is responsible for opening the chromatin for Runx2-driven transcription? Based on our observation that BMP2 treatment increased H3K9me2 (a repressive marker) levels on Runx2 target elements (Figure $6 \mathrm{~F}$ ), we infer that chromatin modifications other than H3K9me2 are likely responsible for the increased Runx2 transcriptional activity during BMP2-induced differentiation, which remain to be studied.

The Runx2-K245 demethylation by PHF2 was identified in MS analysis and further confirmed using K245-mutated Runx2. The SUV39H1-induced methylation of Runx 2 was clearly demonstrated in an in vitro methylation assay, which was abolished by PHF2 (Figure 7C). This result strongly indicates that SUV39H1 and PHF2 target the same lysine residue. Given that PHF2 demethylates K245 of Runx2, SUV39H1 may also methylate the K245 residue, which was further evaluated in Figure 7D. Based on these results, we propose that SU-
V39H1 and PHF2 counterbalance K245 methylation in Runx2. However, the methylation level of K245-mutated Runx2 was still slightly increased upon SUV39H1 overexpression (Figure 7D), suggesting that residues other than K245 might also be involved in SUV39H1-mediated Runx2 methylation.

This study is motivated by previous reports that mutation of the PHF2 gene causes dwarfism in mice [16] and that PHF2 and Runx2 expression are correlated in differentiating thymocytes [17]. Our observation of PHF2-mediated demethylation and activation of Runx 2 provides a theoretical basis for developing therapeutics targeting this demethylase to treat bone development disorders or to accelerate fracture healing.

\section{Materials and Methods}

\section{Osteoblast isolation and cell culture}

Osteoblasts were isolated from calvarial bones of newborn mice. Bones were digested with $0.1 \%$ collagenase (Sigma-Aldrich) and $0.2 \%$ Dispase (Roche, Basel, Switzerland), and cultured in $\alpha$-MEM (without ascorbic acid) supplemented with $10 \%$ fetal bovine serum (FBS). On day 2, cells were reseeded at $1 \times 10^{4}$ per well in a 48 -well dish, and cultured in $\alpha$-MEM containing $10 \%$ FBS, $50 \mathrm{mg} / \mathrm{L}$ of ascorbic acid, $10 \mathrm{mM} \beta$-glycerphosphate, and $50 \mathrm{ng} / \mathrm{ml}$ of BMP2 (Peprotech, Rocky Hill, NJ, USA) to stimulate cell differentiation and maturation. $\mathrm{C} 2 \mathrm{C} 12$ (mouse mesenchymal precursor) and HEK293T (human embryonic kidney) cell lines were obtained from American Type Culture Collection (ATCC, Manassas, VA, USA) and cultured in DMEM supplemented with $10 \% \mathrm{FBS}$.

\section{Plasmids, siRNA and tranfection}

Myc-tagged mouse Runx2 and OG2 reporter plasmids were kindly given by Dr Hyun-Mo Ryoo (Seoul National University School of Dentistry), and Flag-SUV39H1 and Flag-G9a plasmids by Dr Hong-Duk Youn (Seoul National University College of Medicine). The plasmids for Flag/streptavidin binding protein (F/S)-tagged Runx2 fragments were re-cloned from the Runx2 plasmid using $p f u$ polymerase-based PCR and blunt-end ligation.

Figure 8 SUV39H1 negatively regulated osteoblast differentiation. (A) C2C12 cells were transfected with the indicated siRNAs and incubated with BMP2. SUV39H1 knockdown was verified by immunoblotting (top panel). Osteoblast differentiation was monitored by ALP stainingand and Alizarin staining for the indicated times. (B) The expression levels of the osteogenic genes were analyzed by RT-qPCR and presented as relative values to the day- 0 level. Each point represents the mean \pm SD $(n=3)$. * denotes $P<0.05$ vs the si-Con value on the corresponding day. (C) C2C12 cells were transfected with OG2 promoter-luciferase reporter, $\beta$-gal, the indicated siRNAs, and/or Myc-Runx2 plasmid. Each bar represents the mean \pm SD $(n=$ 4) for luciferase activity. (D) C2C12 cells, which had been transfected as indicated, were incubated with BMP2 for $24 \mathrm{~h}$. Each bar represents the mean $\pm \mathrm{SD}(n=4)$, * and n.s. denote $P<0.05$ and non-significant, respectively. The protein levels were immunoblotted (bottom panel). (E-F) C2C12 cells were transfected with SUV39H1 plasmid or siRNA, and incubated with BMP2 for 2 days. ChIP with anti-Runx2 was performed to identify the binding of Runx2 to OCN promoter. Precipitated DNA segments were quantified by real-time PCR. The results (mean $\pm \mathrm{SD}, n=3$ ) are expressed as percentages of the input level. (G) The proposed mechanism underlying Runx2 regulation via lysine methylation. SUV39H1 inhibits the transcriptional activity of Runx2 by methylating Runx2 at Lys 245 [off]. Conversely, PHF2 activates Runx2 by removing the methyl group from Runx2 [on]. PHF2 and SUV39H1 counterbalance osteoblast differentiation by reciprocally regulating Runx2 methylation. 
The cDNA of PHF2 was cloned by RT-PCR with specific primers and amplified using $p f u$ polymerase. The cDNA was inserted into Flag- or F/S-tagged vector. The plasmids for F/S-tagged PHF2 fragments were re-cloned from the PHF2 plasmid using BamHI and EcoRI enzymes. Specific sites of Runx2 and PHF2 were replaced using PCR-based mutagenesis. For gene silencing, control (Sh-Con) or PHF2-targeting shRNA (Sh-PHF2) was inserted into the pLKO.1-puro vector using AgeI and EcoRI enzymes. The viral vector was co-transfected with pMD2-VSVG, pRSV-Rev, and pMDLg/pRRE helper DNA into HEK293T cells, and viral supernatant was collected $48-72 \mathrm{~h}$ later. $\mathrm{C} 2 \mathrm{C} 12$ cells were infected overnight with the virus in the presence of $6 \mu \mathrm{g} / \mathrm{ml}$ of polybrene, and the infected cells were selected with $2 \mu \mathrm{g} / \mathrm{ml}$ of puromycin. For transient transfection of plasmids or siRNAs, cells were transfected with plasmids or siRNAs using Lipofectamine (Invitrogen, Carlsbad, CA, USA). The transfected cells were stabilized for 48 $\mathrm{h}$ before experiments. The sequences of shRNAs and siRNAs are summarized in Supplementary information, Table S1.

\section{Reporter assay}

To evaluate the transcriptional activity of Runx2, two Runx2-responsive luciferase reporter plasmids were used: pGL3-6 $\times$ OSE-luciferase plasmid containing six copies of the Runx2-binding DNA sequence (5'-TGTGGTTGGTGATTGCAGCGGATCTGC-3') and pGL3-OG2-luciferase plasmid containing the proximal segment $(-1$ to $-905 \mathrm{bp}$ ) of the mouse $\mathrm{OCN}$ promoter (NCBI \# MMU66848). $\mathrm{C} 2 \mathrm{C} 12$ cells were cotransfected with the luciferase reporter plasmid and the $\beta$-gal plasmid. The final DNA or siRNA concentration was adjusted by adding pcDNA or control siRNA, respectively. Luciferase activity was assayed using cell extracts by a Lumat LB9507 luminometer (Berthold Technologies, Bad Wildbad, Germany). $\beta$-gal activity was assessed using a spectrophotometer to normalize transfection efficiencies.

\section{ALP and Alizalin Red S stainings.}

For ALP staining, C2C12 cells and primary osteoblasts were cultured in 48-well plates at a density of $1 \times 10^{4}$ cells/well. After differentiation in an osteogenic meduim, cells were fixed with $10 \%$ formaldehyde, rinsed with water, and permeabilized by $0.1 \%$ Triton X-100. Cells were incubated at $37{ }^{\circ} \mathrm{C}$ for $30 \mathrm{~min}$ in the Naphthol-AS-BL alkaline solution mixture (Sigma-Aldrich). For Alizalin Red S staining, cells were cultured in 6-well plates at a density of $1 \times 10^{5}$ cells/well, After being fixed in $3.7 \%$ paraformaldehyde for $10 \mathrm{~min}$, cells were stained with $2 \%$ Alizarin Red S (pH 4.2) for $30 \mathrm{~min}$, rinsed with PBS, and photographed.

\section{Immunoblotting, immunoprecipitation, and streptoavidin pull-down assay \\ Total proteins were separated on $8 \%$ or $12 \%$ SDS/polyacryl-} amide gel and transferred to Immobilon-P membranes. The membranes were preincubated in 5\% non-fat milk for $60 \mathrm{~min}$, and then sequentially incubated with a primary antibody (diluted 1:2501:5 000 in $5 \%$ nonfat milk) overnight at $4{ }^{\circ} \mathrm{C}$ and incubated with a secondary antibody conjugated with horseradish peroxidase (diluted 1:5 000 in 5\% non-fat milk) at room temperature for 60 min. Primary antibodies against PHF2, SUV39H1, H3K9me2, H3K27me2, H3K36me2, H3 were purchased from Cell Signaling (Danvers, MA, USA); anti-Runx2 was from MBL (Woburn, MA, USA); anti-Flag was from Sigma-Aldrich (St Louis, MO, USA);
anti-Runx2, anti-c-Myc, anti-G9a, and anti- $\beta$-tubulin were from Santa Cruz biotechnology (Santa Cruz, CA, USA); anti-methylated lysine and anti-SUV39H1 were from Abcam (Cambridge, UK). For immunoprecipitation, cell lysates ( $1 \mathrm{mg}$ protein) were incubated with $1 \mu \mathrm{g}$ of an antibody for $12 \mathrm{~h}$ and further incubated with 20 $\mu 1$ of protein A/G-sepharose beads (GE Healthcare Bio-Sciences, Piscataway, NJ) for $4 \mathrm{~h}$. The precipitated immune complexes were eluted in a denaturing SDS sample buffer, and then subjected to immunoblotting. For streptoavidin pull-down assay, FlagPHF2 and one of four Flag/SBP-Runx2 fragments or Myc-Runx2 and one of four Flag/SBP-PHF2 fragments were coexpressed in HEK293T cells. Cell lysates ( $1 \mathrm{mg}$ of proteins) were incubated with $10 \mu \mathrm{l} / \mathrm{mg}$ of streptavidin-affinity beads (GE Healthcare Bio-Sciences) at $4{ }^{\circ} \mathrm{C}$ for $3 \mathrm{~h}$. After the beads were washed with the lysis buffer, the pulled-down proteins were eluted in a denaturing SDS sample buffer, and then subjected to western blotting.

\section{$R T-q P C R$}

Total RNA was extracted from cultured cells using Trizol reagent (Invitrogen). cDNAs were synthesized from $1 \mu \mathrm{g}$ of total RNAs using the cDNA synthesis kit provided by Applied Biological Masterials Inc (Richmond, BC). cDNAs were amplified using the ABI StepOne Real-Time PCR (Applied Biological Masterials Inc). Their levels were quantified by calculating $\mathrm{Ct}$ (threshold cycle) values and normalized by $18 \mathrm{~S}$ RNA levels. To examine the temporal changes of mRNA levels during osteoblast differentiation, the results in each group were presented as relative values to the day- 0 value in the corresponding group. The sequences of PCR primers are summarized in Supplementary information, Table S1.

\section{Chromatin immunoprecipitation (ChIP)}

Chromatins were cross-linked with $1 \%$ formaldehyde for 15 min, followed by washing with ice-cold PBS. The cells were collected by scraping and centrifugation, and the pellets were lysed in the FA lysis buffer (50 mM HEPES, pH 7.5, $140 \mathrm{mM} \mathrm{NaCl}, 1$ mM EDTA, $1 \%$ Triton X-100, $0.1 \%$ sodium deoxycholate, $0.1 \%$ SDS, and a protease inhibitor cocktail). The lysates were sonicated to chop chromosomal DNAs into 400-700 bp pieces. The lysates were spun down and diluted by five folds in a chromatin RIPA buffer (50 mM Tris, pH 8.0, $150 \mathrm{mM} \mathrm{NaCl}, 2$ mM EDTA, $1 \%$ NP- $40,0.5 \%$ sodium deoxycholate, $0.1 \%$ SDS, and a protease inhibitor cocktail). The samples were precleaned with protein $\mathrm{A} / \mathrm{G}$ beads, and $1 \%$ of each sample was used as the input control. The samples were immunoprecipitated with anti-Runx2, anti-PHF2, anti-H3K9me2, and anti-Lysine me1/2, or control IgG. The complexs were washed with a buffer ( $20 \mathrm{mM}$ Tris, $\mathrm{pH}$ 8.0, 0.1\% SDS, $1 \%$ Triton X-100, 2 mM EDTA, and $150 \mathrm{mM} \mathrm{NaCl}$ ), and eluted with an elution buffer ( $1 \% \mathrm{SDS}, 100 \mathrm{mM} \mathrm{NaHCO}$ ). Immunoprecipitated DNAs were subjected to PCR amplification using specific primers, whose sequences are summarized in Supplementary information, Table S1, for Runx2-binding elements in the osteocalcin gene.

\section{In vitro methylation assay}

Recombinant GST-tagged Runt domain peptide (GST-R2) was expressed in E. coli using IPTG and purified using GSH-affinity beads. Flag-SUV39H1 and Flag/SBP-PHF2 peptides were expressed in HEK293T cells and purified by affinity chromatography using EZview ${ }^{\mathrm{TM}}$ Red anti-FALG ${ }^{\circledR}$ M2 beads and Flag peptide (Sig- 
ma-Aldrich). To methylate the Runt domain in vitro, GST-R2 (1 $\mu \mathrm{g}$ per reaction) was incubated with $50 \mu \mathrm{M}$ S-adenosyl methionine (SAM), Flag-SUV39H1 (500 ng), and/or Flag/SBP-PHF2 (250 $\mathrm{ng}$ ) in the methylation buffer containing $50 \mathrm{mM}$ Tris/ $\mathrm{HCl}(\mathrm{pH} \mathrm{8.0})$, $150 \mathrm{mM} \mathrm{NaCl}, 1 \mathrm{mM}$ EDTA, $0.02 \%$ Triton X-100, and protease inhibitor cocktail. The reaction was incubated for $60 \mathrm{~min}$ at $37{ }^{\circ} \mathrm{C}$ or at $4{ }^{\circ} \mathrm{C}$ and stopped by boiling in a SDS loading buffer. The reaction mixtures were eletrophoresed and the methylation of GST$\mathrm{Rx} 2$ was detected using anti-methylated lysine antibody.

\section{Immunofluorescence and immunohistochemistry}

For immunofluorescence, cells on coverslips were fixed with $3.7 \%$ paraformaldehyde and permeabilized with $0.1 \%$ Triton $\mathrm{X}-100$. Non-specific protein binding was blocked with $1 \%$ goat serum and cells were incubated with primary antibody overnight at $4{ }^{\circ} \mathrm{C}$. After brief washing, cells were incubated with a fluorescence-probe-conjugated secondary antibody for $1 \mathrm{~h}$. Excised calvarias were fixed with $3.7 \%$ paraformaldehyde and embedded in paraffin. Serial sections $(4 \mu \mathrm{m})$ were cut from each block, deparaffinized, rehydrated in a graded alcohol series, and heated in a microwave for $15 \mathrm{~min}$ in $10 \mathrm{mM}$ sodium citrate ( $\mathrm{pH} \mathrm{6.0)}$ ) to retrieve antigens. Sections were sequentially incubated with blocking solution for $1 \mathrm{~h}$, with anti-PHF2 or anti-Flag antibody overnight at $4{ }^{\circ} \mathrm{C}$, and Alexa 586-conjugated anti-rabbit or biotinylated secondary (for anti-Flag stained slides) antibody (Invitrogen) for $1 \mathrm{~h}$. Stained cells and tissues were mounted on glass slides to evaluate signal under a fluorescence microscope (Olympus). For immunohistochemistry, the mouse calvarial sections were sequentially incubated with a blocking solution for $1 \mathrm{~h}$ and with the anti-Flag antibody overnight at $4{ }^{\circ} \mathrm{C}$. The slides were incubated with biotinylated secondary antibody for $2 \mathrm{~h}$, and then visualized using the ABC kit (VECTOR, Burlingame, CA, USA) and DAB (DAKO, Denmark).

\section{Generation of PHF2 transgenic mice and skeletal staining.}

Transgenic mice were created by injecting the CMV-FlagPHF2 vector into fertilized eggs from C57BL6 mice. Transgenic lines were established from 9 founders that were identified via PCR-based genotyping, and a transgenic line was used in our experiments. For genotyping, genomic DNAs extracted from tail biopsies were amplified using transgene-specific PCR primers. Hemizygote males were bred with wild-type females to produce hemizygous transgenic and wild-type littermates. All experiments were done in compliance with the guide included in the Seoul National University Laboratory Animal Maintenance Manual (approve No. SNU-100805-2-2). For bone and cartilage staining, newborn mice were eviscerated, fixed in $95 \%$ ethanol for 1 day, and transferred to acetone. After 3 days, the samples were rinsed with water and stained for 2 days with $0.005 \%$ Alizarin red S and $0.015 \%$ Alcian blue $8 \mathrm{GX}$ in ethanol. After rinsing with water, the samples were kept in $20 \%$ glycerol $/ 1 \% \mathrm{KOH}$ until the skeletons became clearly visible. For storage, they were serially transferred into $50 \%, 80 \%$, and $100 \%$ glycerol. All reagents used were purchased from Sigma-Aldrich.

\section{Calcein-Alizarin double staining}

After heterozygous PHF2-t/g female mice had mated with wildtype male mice, pregnant mice having wild-type and PHF2-t/g fetuses were intraperitoneally injected with fluorochrome-labeled
Calcein green $(10 \mathrm{mg} / \mathrm{kg}$, Sigma-Aldrich) on day 16.5 of pregnancy. Two days later, the pregnant mice were secondarily injected with Alizarin complexone (50 mg/kg, Sigma-Aldrich, USA). On postnatal day 1 ( 2 days after the second injection), tails of newborn mice were biopsied for genotyping and calvarias were fixed in $70 \%$ ethanol. Calcein- and Alizalin-labeled calvarias were examined under a fluorescence microscope.

\section{von Kossa staining.}

Mouse calvarias were fixed with $3.7 \%$ paraformaldehyde and embedded in Tecnovit 7200 resin. Tissue sections $(50 \mu \mathrm{m})$ were cut, incubated in 5\% silver nitrate for $1 \mathrm{~h}$, and exposed to bright light for $15 \mathrm{~min}$. Sections were then incubated in 5\% sodium thiosulfate for $3 \mathrm{~min}$, rinsed in water, and counterstained with Nuclear Fast Red.

\section{Surgery for generating calvarial defects in rats.}

To study bone regeneration, we produced calvarial defects in 10-week-old, male SD rats weighing 300-350 g (Orient, Gyeonggki-do, Korea). A semilunar incision was made in the scalp to allow reflection of a full-thickness flap. We prepared an 8-mm-diameter bone defect in calvarias with a trephine bur (GEBR, Brasseler, Germany). The calvarias were covered with a collagen matrix (Cowellmedi Co., Busan, Korea) containing $2 \mathrm{mg}$ of BMP-2 and $5 \times 10 \% / \mathrm{ml}$ of shRNA lentiviruses, and skin flaps were sutured. Eight rats per each group were sacrificed 8 weeks after surgery. For histological analysis, calvarial samples were fixed in $4 \%$ paraformaldehyde for $24 \mathrm{~h}$, decalcified with 10\% EDTA for 10-14 days, embedded in paraffin, and sectioned at $4 \mu \mathrm{m}$. Sections were stained with Hematoxylin and Eosin for histological analysis. The procedures used and the care of animals were in accordance with the guide included in the Seoul National University Laboratory Animal Maintenance Manual (approve No. SNU-121214-2-1)

\section{Micro-computed tomography (micro-CT).}

Micro-CT of rat calvaria was performed using a Skyscan 1172 scanner (Bruker-microCT, Kontich, Belgium). Scanning was carried out at $70 \mathrm{kV} / 141 \mu \mathrm{A}$ for 1180 milliseconds. In total, 1004 projections were collected at a resolution of $14.87 \mu \mathrm{m} /$ pixel. Reconstruction of sections was carried out with software associated with the scanner (Nrecon) with beam hardening correction set to 5\%. Realistic 3D-Visulizatio software (Bruker-microCT, Konitch, Belgium) was used to reconstruct the CT images 3-dimensionally. Micro-CT of mice whole-body was taken with a NFR Polaris-G90 (Nanofocusray, Jeonju, Korea). Scanning was carried out at 65 $\mathrm{kV} / 120 \mu \mathrm{A}$ for 150 milliseconds. Data were acquired from 1024 images of reconstruction with an isotropic voxel spacing of 0.034 $\times 0.034 \times 0.027 \mathrm{~mm}^{3}$. The volume of bone ingrown in a defect site was analyzed 3-dimensionally using a software Amira version 5.4.1 (Visage Imaging GmbH, Berlin, Germany).

\section{In-gel digestion and MS analysis}

Flag/SBP-R2 peptide expressed in $\mathrm{C} 2 \mathrm{C} 12$ stable cell lines were bound to Flag-affinity beads $\left(10 \mu \mathrm{l} / \mathrm{mg}\right.$ protein) for $4 \mathrm{~h}$ at $4{ }^{\circ} \mathrm{C}$ and eluted with Flag peptide $(0.2 \mu \mathrm{g} / \mathrm{ml})$. The eluted Flag/SBP-R2 peptide was secondarily bound to streptavidin beads $(10 \mu \mathrm{l} / \mathrm{mg})$ for $1 \mathrm{~h}$ at $4{ }^{\circ} \mathrm{C}$, eluted with a denaturing SDS sample buffer, and subjected to SDS-PAGE analysis. For in-gel digestion, gel pieces were reduced at $56{ }^{\circ} \mathrm{C}$ for $45 \mathrm{~min}$ by $10 \mathrm{mM}$ DTT, followed by al- 
kylation of cysteines with $55 \mathrm{mM}$ iodoacetamide for $30 \mathrm{~min}$ in the dark. Gel pieces were treated overnight with $12.5 \mathrm{ng} / \mathrm{l}$ of trypsin (Promega, Madison, WI) in $50 \mathrm{mM} \mathrm{NH}_{4} \mathrm{HCO}_{3}$ buffer (pH 7.8) at $37{ }^{\circ} \mathrm{C}$. Digested peptides were extracted with $5 \%$ formic acid for $20 \mathrm{~min}$, and the supernatants were dried using SpeedVac. Samples suspended in $0.1 \%$ formic acid were purified and concentrated using C18 ZipTips (Millipore, MA) before MS analysis. The peptides were loaded onto a fused silica microcapillary column (12 $\mathrm{cm} \times 75 \mu \mathrm{m})$ packed with $\mathrm{C} 18$ reversed phase resin $(5 \mu \mathrm{m}, 200$ $\&)$. LC separation was conducted under a linear gradient as follows: a 3\%-40\% solvent (acetonitrile containing $0.1 \%$ formic acid) gradient with a flow rate of $250 \mathrm{nl} / \mathrm{min}$ for $60 \mathrm{~min}$. The column was directly connected to LTQ linear ion-trap mass spectrometer (Finnigan, CA) equipped with a nano-electrospray ion source. The electrospray voltage was set at $1.95 \mathrm{kV}$, and the threshold for switching from MS to MS/MS was 500. The normalized collision energy for MS/MS was $35 \%$ of main radio frequency amplitude and the duration of activation was 30 milliseconds. All spectra were acquired in data-dependent scan mode. Each full MS scan was followed by five MS/MS scan corresponding from the most intense to the fifth intense peaks of full MS scan. Repeat count of peak for dynamic exclusion was 1 , and its repeat duration was 30 $\mathrm{s}$. The dynamic exclusion duration was set for $180 \mathrm{~s}$ and width of exclusion mass was $\pm 1.5 \mathrm{Da}$. The acquired spectra were searched in the BioWorksBrowser ${ }^{\mathrm{TM}}$ (version Rev. 3.3.1 SP1, Thermo Fisher Scientific Inc., CA) with the SEQUEST search engines against non-redundant human database on National Center for Biotechnology Information (http://www.ncbi.nlm.nih.gov/).

\section{Statistical analysis}

All data were analyzed using Microsoft Excel 2010 software, and results are expressed as means and standard deviations. We used the unpaired, two-sided Student $t$-test to compare reporter activities and morphometric data from micro-CT analyses. Statistical significances were considered when $P<0.05$.

\section{Acknowledgments}

This work was supported by grants from the Bone Metabolism Research Center (SRC; 2011-0001026), the Korean Health Technology R\&D (A121106 and A120476), and the National Research Foundation (2009-0090188, 2011-0030737 and 2013R1A2A1A01015228) supported by Korean government.

\section{References}

1 Fortschegger K, Shiekhattar R. Plant homeodomain fingers form a helping hand for transcription. Epigenetics 2011; 6:4-8.

2 Nakayama J, Rice JC, Strahl BD, Allis CD, Grewal SI. Role of histone $\mathrm{H} 3$ lysine 9 methylation in epigenetic control of heterochromatin assembly. Science 2001; 292:110-113.

3 Vakoc CR, Mandat SA, Olenchock BA, Blobel GA. Histone H3 lysine 9 methylation and HP1gamma are associated with transcription elongation through mammalian chromatin. Mol Cell 2005; 19:381-391.

4 Baba A, Ohtake F, Okuno Y, et al. PKA-dependent regulation of the histone lysine demethylase complex PHF2-ARID5B. Nat Cell Biol 2011; 13:668-675.

5 Okuno Y, Ohtake F, Igarashi K, et al. Epigenetic regulation of adipogenesis by phf2 histone demethylase. Diabetes 2013; 62:1426-1434.

6 Stender Joshua D, Pascual G, et al. Control of proinflammatory gene programs by regulated trimethylation and demethylation of histone H4K20. Mol Cell 2012; 48:28-38.

7 Schroeder TM, Jensen ED, Westendorf JJ. Runx2: a master organizer of gene transcription in developing and maturing osteoblasts. Birth Defects Res C Embryo Today 2005; 75:213225.

8 Long F. Building strong bones: molecular regulation of the osteoblast lineage. Nat Rev Mol Cell Biol 2012; 13: 27-38.

9 Kugimiya F, Kawaguchi H, Ohba S, et al. GSK-3beta controls osteogenesis through regulating Runx2 activity. PLoS One 2007; 2:e837.

10 Jonason JH, Xiao G, Zhang M, Xing L, Chen D. Post-translational regulation of Runx 2 in bone and cartilage. J Dent Res 2009; 88:693-703.

11 Jun JH, Yoon WJ, Seo SB, et al. BMP2-activated Erk/MAP kinase stabilizes Runx2 by increasing p300 levels and histone acetyltransferase activity. J Biol Chem 2010; 285:3641036419.

12 Zhao M, Qiao M, Oyajobi BO, Mundy GR, Chen D. E3 ubiquitin ligase Smurfl mediates core-binding factor alpha1/ Runx2 degradation and plays a specific role in osteoblast differentiation. J Biol Chem 2003; 278:27939-27944.

13 Schroeder TM, Kahler RA, Li X, Westendorf JJ. Histone deacetylase 3 interacts with runx 2 to repress the osteocalcin promoter and regulate osteoblast differentiation. $\mathrm{J} \mathrm{Biol} \mathrm{Chem}$ 2004; 279:41998-42007.

14 Bradley EW, McGee-Lawrence ME, Westendorf JJ. Hdac-mediated control of endochondral and intramembranous ossification. Crit Rev Eukaryot Gene Expr 2011; 21:101-113.

15 Yang D, Okamura H, Nakashima Y, Haneji T. Histone demethylase Jmjd3 regulates osteoblast differentiation via transcription factors Runx2 and Osterix. J Biol Chem 2013; 288:33530-33541.

16 Hansen J, Floss T, Van Sloun P, et al. A large-scale, gene-driven mutagenesis approach for the functional analysis of the mouse genome. Proc Natl Acad Sci USA 2003; 100:99189922.

17 Zhao FQ, Sheng ZM, Tsai MM, et al. Serial analysis of gene expression in murine fetal thymocyte cell lines. Int Immunol 2002; 14:1383-1395.

18 Huang J, Berger SL. The emerging field of dynamic lysine methylation of non-histone proteins. Curr Opin Genet Dev 2008; 18:152-158.

19 Shinkai Y, Tachibana M. H3K9 methyltransferase G9a and the related molecule GLP. Genes Dev 2011; 25:781-788.

20 Rathert P, Dhayalan A, Murakami M, et al. Protein lysine methyltransferase G9a acts on non-histone targets. Nat Chem Biol 2008; 4:344-346.

21 Jonason JH, Xiao G, Zhang M, Xing L, Chen D. Post-translational regulation of Runx 2 in bone and cartilage. J Dent Res 2009; 88:693-703.

22 Kim BG, Kim HJ, Park HJ, et al. Runx2 phosphorylation induced by fibroblast growth factor-2/protein kinase $\mathrm{C}$ pathways. Proteomics 2006; 6:1166-1174.

23 Selvamurugan N, Pulumati MR, Tyson DR, Partridge NC. Parathyroid hormone regulation of the rat collagenase-3 pro- 
moter by protein kinase A-dependent transactivation of core binding factor alpha1. J Biol Chem 2000; 275:5037-5042.

24 Wee HJ, Huang G, Shigesada K, Ito Y. Serine phosphorylation of RUNX2 with novel potential functions as negative regulatory mechanisms. EMBO Rep 2002; 3:967-974.

25 Jeon EJ, Lee KY, Choi NS, et al. Bone morphogenetic protein-2 stimulates Runx2 acetylation. J Biol Chem 2006; 281:16502-16511.

26 Huang J, Dorsey J, Chuikov S, et al. G9a and Glp methylate lysine 373 in the tumor suppressor p53. J Biol Chem 2010; 285:9636-9641.

27 Lee JS, Kim Y, Kim IS, et al. Negative regulation of hypoxic responses via induced Reptin methylation. Mol Cell 2010; 39:71-85.

28 Chakraborty S, Sinha KK, Senyuk V, Nucifora G. SUV39H1 interacts with AML1 and abrogates AML1 transactivity. AML1 is methylated in vivo. Oncogene 2003; 22:5229-5237.

29 Reed-Inderbitzin E, Moreno-Miralles I, Vanden-Eynden SK, et al. RUNX1 associates with histone deacetylases and SUV39H1 to repress transcription. Oncogene 2006; 25:57775786.

30 Tandon M, Gokul K, Ali SA, et al. Runx2 mediates epigenetic silencing of the bone morphogenetic protein-3B (BMP-3B/ GDF10) in lung cancer cells. Mol Cancer 2012; 11:27.

(Supplementary information is linked to the online version of the paper on the Cell Research website.) 